\title{
Structural, vibrational, and dynamic properties of Ge-Ga-Te liquids with increasing connectivity: A combined neutron scattering and molecular dynamics study
}

\author{
H. Flores-Ruiz, ${ }^{1,2}$ M. Micoulaut,,${ }^{1, *}$ A. Piarristeguy, ${ }^{3}$ M.-V. Coulet, ${ }^{4}$ M. Johnson, ${ }^{5}$ G. J. Cuello, ${ }^{5}$ and A. Pradel ${ }^{3}$ \\ ${ }^{1}$ Sorbonne Université, Physique Théorique de la Matière Condensée, UMR 7600, 75252 Paris Cedex 05, France \\ ${ }^{2}$ Departamento de Ciencias Naturales y Exactas, CUValles, Universidad de Guadalajara, Carr. Guadalajara-Ameca km 45.5, 46600, \\ Ameca, Jalisco. México \\ ${ }^{3}$ Institut Charles Gerhardt, UMR 5253-CNRS, Case 1503, Université de Montpellier, Place Eugène Bataillon, \\ 34095 Montpellier Cedex 5, France \\ ${ }^{4}$ Aix-Marseille Université, CNRS, MADIREL UMR 7246, Campus de Saint Jérôme, 13397 Marseille Cedex 20, France \\ ${ }^{5}$ Institut Laue Langevin, 71 Avenue des Martyrs, 38042 Grenoble, France
}

(Received 28 February 2018; revised manuscript received 28 May 2018; published 26 June 2018)

\begin{abstract}
The physical properties of several compositions of $\mathrm{Ge}_{x} \mathrm{Ga}_{x} \mathrm{Te}_{100-2 x}$ liquids $(x=6,10,14 \%)$ are studied using a combination of density functional based molecular dynamics simulations and neutron experiments. We investigate structural properties including structure factors, pair distribution functions, angular distributions, coordination numbers, neighbor distributions and compare the results with diffraction experiments. We find that $\mathrm{Ga}$ is essentially in tetrahedral configuration and also affects the tetrahedral character of germanium when the results are compared to similar telluride liquids, while Te atoms display a coordination number that is larger than 2 and depend substantially on composition. The vibrational density of states is also measured from inelastic neutron scattering and compared to the simulated counterpart which exhibits a very good agreement at low frequency. We finally determine the dynamics of the melts by evaluating the diffusivity and relaxation properties. These reveal that Ge-Ga-Te melts are extremely fragile $(\mathcal{M} \simeq 139)$, similarly to Ge-Sb-Te liquids.
\end{abstract}

DOI: 10.1103/PhysRevB.97.214207

\section{INTRODUCTION}

Compared to the parent system Ge-Sb-Te now used in phase change (PC) applications [1], amorphous gallium based tellurides have received much less attention. However, recent investigations suggest that such materials also display interesting optoelectronic properties and might therefore also represent commercially attractive materials. Indeed, the addition of $\mathrm{Ga}$ or $\mathrm{Ge}$ into a Te-rich alloy has been found to increase the thermal stability of the amorphous phase and the speed of crystallization [2], the latter representing a crucial property for PC materials that needs to be continuously optimized.

Ga-based tellurides have received increased attention in recent years due to their remarkable transparency up to $20 \mu \mathrm{m}$ as many other Te-based materials. Some applications require microcomponents being able to work in an extended infrared domain, like spatial interferometry, biosensing, or environmental metrology. Different Te-based systems are particularly attractive in this perspective, such as Te-Ge-I [3], Te-As-Se [4,5], and the Ga-based amorphous Ge-Ga-Te [6-8] and GaAs-Se-Te [9]. After purification of the elemental precursors, it was shown for example that the $\mathrm{Ge}_{15} \mathrm{Ga}_{10} \mathrm{Te}_{75}$ bulk glass transmits light from 4 up to $20 \mu \mathrm{m}$ [8], which makes it a promising candidate for spatial interferometry. The main drawback is the strong crystallization tendency that limits the bulk glass-forming domain by conventional quenching methods to compositions close to the $\mathrm{GeTe}_{4}-\mathrm{GaTe}_{3}$ join in the $\mathrm{Ga}-\mathrm{Ge}-\mathrm{Te}$

*mmi@1ptmc.jussieu.fr
(GGT) ternary [6,8]. However, alternative methods (thermal co-evaporation, twin-roller quenching) have permitted us to extend this domain for very thick films.

The amorphous structure of the Ge-Ga-Te system is partially unknown, although recent theoretical and experimental studies [10] for a select composition $\left(\mathrm{Ge}_{11} \mathrm{Ga}_{11} \mathrm{Te}_{78}\right)$ have emphasized that the fourfold coordination of $\mathrm{Ga}$ is tetrahedral, while Ge has an increased tendency to form tetrahedral configurations as compared to the Ge-Sb-Te system. Such results are partially recovered from extended x-ray absorption fine structure experiments (EXAFS) spectroscopies and reverse Monte Carlo modeling [11,12], and these have been linked with kinetic aspects [13].

In the present contribution, we determine the structural and vibrational properties in the Ge-Ga-Te liquid alloy by investigating the combined addition of $\mathrm{Ge}$ and $\mathrm{Ga}$ in a base Te liquid. We perform neutron experiments and density functional (DFT) based molecular dynamics (MD) simulations. Corresponding structure factors and pair correlation functions are reproduced and the agreement of the calculation with experiments is found to increase as the Te content reduces. In general, Gabased liquids display an increased tendency to form tetrahedral geometries, and this tendency is also dependent on the Te-content, the Ga-rich composition $\left(\mathrm{Ge}_{14} \mathrm{Ga}_{14} \mathrm{Te}_{72}\right)$ having the largest amount $(23.8 \%)$ that is determined from angular topological constraint counting. We then characterize coordination numbers, bond angle distributions before investigating the vibrational density of states both from the experimental and theoretical viewpoint. Finally, we concentrate on the liquid dynamics and calculate diffusion constants. From the 
calculated temperature dependence of the relaxation time, we determine that Ge-Ga-Te liquids are extremely fragile, similarly to Sb-based tellurides [14], with an approximate fragility of $\mathcal{M}=139$ for the $\mathrm{Ge}_{14} \mathrm{Ga}_{14} \mathrm{Te}_{72}$ composition. This high fragility value suggests that the present tellurides, as many others previously investigated [15-17], should also display a fast crystallization behavior at high temperature due to rather low activation barriers for diffusion.

\section{METHODS}

\section{A. Experimental details}

Three Ge-Ga-Te samples of nominal compositions $\mathrm{Ge}_{5.9} \mathrm{Ga}_{5.9} \mathrm{Te}_{88.2}$ (called $\mathrm{Ge}_{6} \mathrm{Ga}_{6} \mathrm{Te}_{88}$ hereafter), $\mathrm{Ge}_{10} \mathrm{Ga}_{10} \mathrm{Te}_{80}$ and $\mathrm{Ge}_{14.3} \mathrm{Ga}_{14.3} \mathrm{Te}_{71.4}$ (called $\mathrm{Ge}_{14} \mathrm{Ga}_{14} \mathrm{Te}_{72}$ hereafter) were used for both neutron diffraction and inelastic scattering experiments. Starting elements from high-purity, germanium pellets (99.999\%, Goodfellow), gallium ingots (99.9995\%, SigmaAldrich), and tellurium ingots (99.9999\%, Sigma-Aldrich) were first weighed in stoichiometric quantities (for a total batch of $\sim 10 \mathrm{~g})$ and introduced in a cylindrical silica tube $(11 \mathrm{~mm}$ inner diameter, $1 \mathrm{~mm}$ thick). The tube was subsequently evacuated under secondary vacuum $\left(10^{-5} \mathrm{mbar}\right)$, sealed and melted at $1220 \mathrm{~K}$ in a furnace with a low heating rate of $10 \mathrm{~K} / \mathrm{h}$. The batch was held at this temperature for three days and cooled down to room temperature with an annealing step of two days at $1073 \mathrm{~K}$.

Neutron diffraction experiments [18] were performed on D4C instrument [19] of the high-flux reactor at the Institute Laue Langevin (ILL, Grenoble, France). The incident neutron wavelength was fixed at $0.4978 \AA$ using the $\mathrm{Cu}(220) \mathrm{Bragg}$ reflection. The scattered intensities were measured through an ensemble of nine moving detector banks (each of 64 cells) giving access to a $k=4 \pi \sin (2 \theta) / \lambda$ range of [0.4-23.5] $\AA^{-1}$ with $2 \theta$ the scattering angle. The total structure factors $S_{T}(k)$ were obtained after standard correction for background and container scattering, self-attenuation, multiple scattering, and inelasticity effects. The program CORRECT [20] was used to perform the analysis. Since no liquid density values are available for those compositions, they were first calculated from the pure elements assuming zero excess volume of mixing. They were afterward adjusted by using the asymptotic limit of the mean differential scattering cross section per atom that must be equal to the theoretical ones within a few percent. The densities obtained for each composition are given in Table I.

Inelastic neutron scattering experiments [18] were carried out on an IN6 spectrometer at a wavelength of $4.14 \AA$. Standard corrections were applied to the data: normalization of the spectra to identical values, subtraction of the container contribution, and normalization to a reference spectrum of a $11 \mathrm{~mm}$ diameter vanadium cylinder. The data were corrected for the energy dependent detector efficiency and time independent background. All the correction procedure was done using the LAMP program [22] using predefined functions. A dynamical structure factor $S(2 \theta, \omega)$ was then obtained for each composition. The structure factor $S(k)$ and the VDOS $g(\omega)$ were afterward obtained by integrating $S(2 \theta, \omega)$, respectively, over the accessible $\omega$ range or the accessible $2 \theta$ range.

For both experiments, a vanadium resistor was used as a furnace. The temperature accuracy was estimated using the melting of each alloy, which can be easily identified owing to the disappearance of Bragg peaks. The investigated thermodynamic conditions were the same for the two experiments and Table I summarizes the chosen compositions and temperatures.

\section{B. Molecular dynamics}

Three compositions of $\mathrm{Ge}_{x} \mathrm{Ga}_{x} \mathrm{Te}_{100-x}(x=6,10,14 \%)$ have been also investigated from MD simulations performed in NVT ensemble. In addition, we have also considered results of elemental liquid $\mathrm{Te}(x=0)$ that have been recently published [23]. The systems contained 300 atoms (Fig. 1) positioned in a periodically repeated cubic cell whose size allows recovering the measured experimental densities $\rho_{\text {expt }}$ reported in Table I and corresponding at $823 \mathrm{~K}$ to a cell length of $21.26 \AA$, $21.64 \AA$, and $21.45 \AA$ for the 6,10 , and $14 \%$ compositions, respectively. The electronic structure was described within DFT, and evolved self-consistently during the motion [24]. A generalized gradient approximation (GGA) has been used, based on an improved scheme for the exchange-correlation energy obtained by Perdew, Burke, and Ernzerhof (PBEsol). This functional has been found to improve substantially the structural description of elemental tellurium in the liquid phase [25] and has also led to an accurate description of $\mathrm{Ga} / \mathrm{Sb}$ films [26]. Alternative exchange-correlation functionals can be used to investigate such chalcogenide glass-forming liquids. While it is now well established that the Becke, Lee, Yang,

TABLE I. Experimental conditions (melting temperature $\mathrm{T}_{m}$, measurement temperatures $\mathrm{T}_{\text {expt }}$, and estimated densities $\rho_{\text {expt }}$ ) in different GGT liquids: $\mathrm{Ge}_{6} \mathrm{Ga}_{6} \mathrm{Te}_{88}, \mathrm{Ge}_{10} \mathrm{Ga}_{10} \mathrm{Te}_{80}$, and $\mathrm{Ge}_{14} \mathrm{Ga}_{14} \mathrm{Te}_{72}$. The others parameters are: measured positions $k_{1}$ and $k_{2}$ of the two first principal peaks of the total structure factors $S_{T}(k)$, order parameter $S=S_{T}\left(k_{2}\right) / S_{T}\left(k_{1}\right)$, first and second neighbor peak positions $r_{1}^{\text {expt }}$ and $r_{2}^{\text {expt }}$ of the total pair correlation function $g(r)$, first minimum position $r_{\min }$ of $g(r)$ and corresponding value $g\left(r_{\min }\right)$ at this first minimum, coordination number $N_{\text {expt }}^{\text {tot }}$ using the minimum $r_{\min }$ of the experimental pair correlation function. $\mathrm{T}_{\text {th }}$ is the temperature at which the structural analysis from MD simulations have been performed and the two last columns give the calculated first and second neighbor peak positions $r_{1}^{\text {th }}$ and $r_{2}^{\text {th }}$ of the total simulated pair correlation function.

\begin{tabular}{|c|c|c|c|c|c|c|c|c|c|c|c|c|c|c|}
\hline & $\begin{array}{l}\mathrm{T}_{m} \\
(\mathrm{~K})\end{array}$ & $\begin{array}{c}\mathrm{T}_{\text {expt }} \\
(\mathrm{K})\end{array}$ & $\begin{array}{l}\mathrm{T}_{\mathrm{th}} \\
(\mathrm{K})\end{array}$ & $\begin{array}{c}\rho_{\text {expt }} \\
\left(\AA^{-3}\right)\end{array}$ & $\begin{array}{c}k_{1} \\
\left(\AA^{-1}\right)\end{array}$ & $\begin{array}{c}k_{2} \\
\left(\AA^{-1}\right)\end{array}$ & $S$ & $\begin{array}{l}r_{1}^{\text {expt }} \\
(\AA)\end{array}$ & $\begin{array}{l}r_{\min } \\
(\AA)\end{array}$ & $g\left(r_{\min }\right)$ & $\begin{array}{l}r_{2}^{\text {expt }} \\
(\AA)\end{array}$ & $N_{\text {expt }}^{\mathrm{tot}}$ & $\begin{array}{l}r_{1}^{\text {th }} \\
(\AA)\end{array}$ & $\begin{array}{l}r_{2}^{\text {th }} \\
(\AA)\end{array}$ \\
\hline $\mathrm{Te}$ & 722 & $722[25]$ & 823 & $0.0270(0)[21]$ & & & & $2.82(5)[25]$ & $3.14(5)[25]$ & $1.02[25]$ & $4.06(5)[25]$ & & $2.91[23]$ & 4.11 [23] \\
\hline $\mathrm{Ge}_{6} \mathrm{Ga}_{6} \mathrm{Te}_{88}$ & 680 & 913 & 913 & $0.0312(4)$ & 2.09 & 3.22 & $0.90(2)$ & $2.75(1)$ & $3.27(1)$ & 0.76 & $4.10(1)$ & $3.48(4)$ & $2.86(0)$ & $4.11(2)$ \\
\hline $\mathrm{Ge}_{10} \mathrm{Ga}_{10} \mathrm{Te}_{80}$ & 640 & 873 & 823 & $0.0296(4)$ & 2.09 & 3.26 & $0.99(3)$ & 2.71(1) & $3.22(1)$ & 0.60 & $4.15(1)$ & $3.21(4)$ & $2.76(1)$ & $4.22(2)$ \\
\hline $\mathrm{Ge}_{14} \mathrm{Ga}_{14} \mathrm{Te}_{72}$ & 690 & 923 & 923 & $0.0304(4)$ & 2.09 & 3.25 & $1.06(1)$ & $2.70(1)$ & $3.25(1)$ & 0.56 & $4.08(1)$ & $3.48(4)$ & $2.70(2)$ & $4.18(4)$ \\
\hline
\end{tabular}




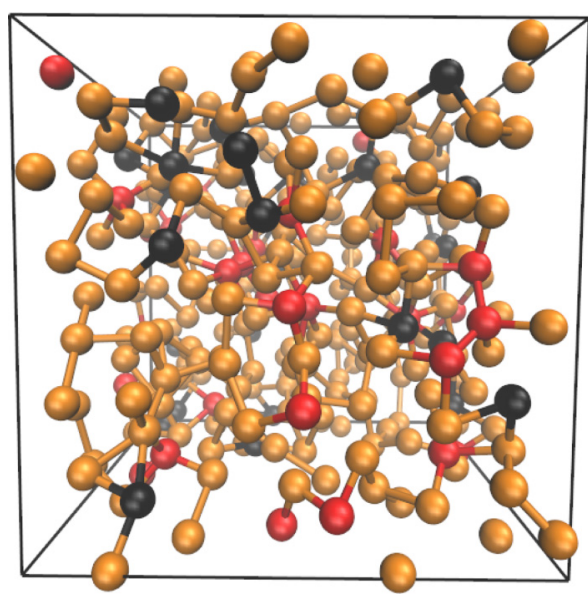

FIG. 1. A typical structure of liquid (823 K) $\mathrm{Ge}_{10} \mathrm{Ga}_{10} \mathrm{Te}_{80}$. Ge, $\mathrm{Ga}$, and $\mathrm{Te}$ atoms are in red, black, and orange, respectively.

and Parr (BLYP) functional improves substantially the liquid structure of lighter chalcogenides (e.g., $\mathrm{GeSe}_{2}$ [27] ), it has been only recently demonstrated that this conclusion might apply to tellurides as well. Massobrio and collaborators have shown, indeed, that BLYP (eventually corrected by dispersion forces) could accurately reproduce neutron structure functions in amorphous $\mathrm{GeTe}_{4}$ [28] and $\mathrm{Ge}_{2} \mathrm{Sb}_{2} \mathrm{Te}_{5}$ [29]. This conclusion remains debated, however, given the opposite conclusion obtained in liquid Tellurium [25]. In the liquid phase of chalcogenides, since one does observe an increased metallic character of the bonding and for the present tellurides an increased $\mathrm{p}$ bonding arising from octahedral germanium, even $\mathrm{GeTe}_{4}$ might be more appropriately described from a DFT recipe building on the uniform electron gas character of the correlation energy such as PBE-based functionals. In terms of methodology, it would certainly be interesting to probe such functionals in a systematic fashion on, e.g., Ga-based tellurides, but this question is clearly out of the scope of the present contribution.

Valence electrons were treated explicitly, in conjunction with Trouiller-Martins norm conserving pseudopotentials [30]. The wave functions have been expanded at the $\Gamma$ point of the supercell on a plane wave basis set with an energy cutoff $E_{c}=$ 20 Ry. During the Car-Parrinello molecular dynamics (CPMD) simulation, a fictitious electron mass of 3000 a.u. and a time step of $\Delta t=0.12 \mathrm{fs}$ have been used to integrate the equations of motion. Temperature control has been implemented for both the ionic and electronic degrees of freedom by using NoséHoover thermostats [31] with a frequency of $800 \mathrm{~cm}^{-1}$ and $3000 \mathrm{~cm}^{-1}$ for the ions and the electronic degrees of freedom, respectively. The simulations have been carried out without dispersion forces, i.e., we did not use the DFT-D2 scheme as in previous studies [23]. Work in this direction is in progress and the present study might be useful as a benchmark for further numerical studies regarding the DFT methodology of tellurides.

The starting configuration of all liquids is a random structure of $\mathrm{Ge}, \mathrm{Ga}$, and $\mathrm{Te}$ atoms fulfilling the desired stoichiometry, and loss of the memory of the initial configurations has been achieved through preliminary runs at $2000 \mathrm{~K}$ and $1500 \mathrm{~K}$ over $50 \mathrm{ps}$. The total simulation time of these preliminary leads to mean square displacements of the order of several cell lengths

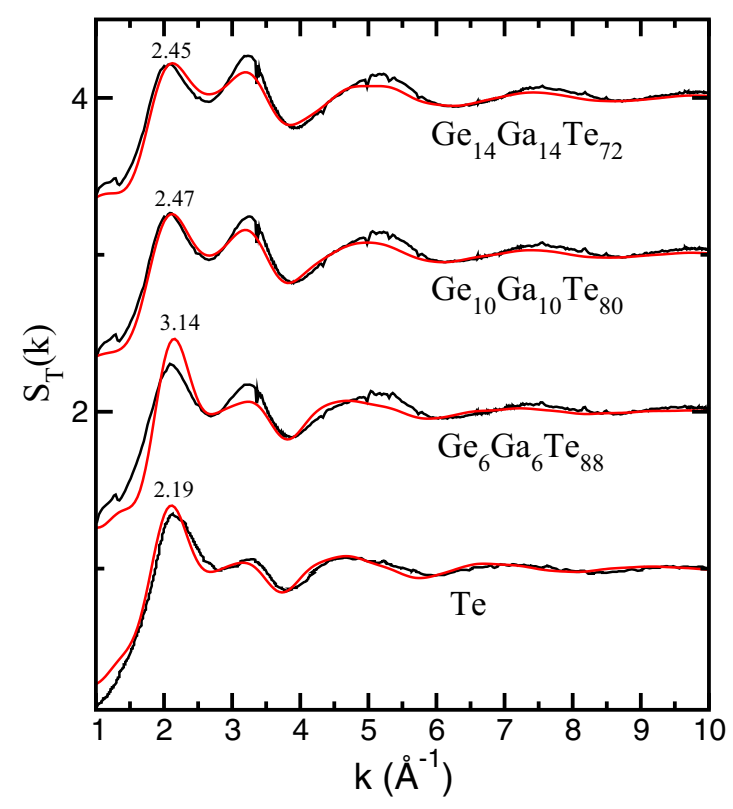

FIG. 2. Evolution of the total structure factor $S_{T}(k)$ in $\mathrm{Ge}_{x} \mathrm{Ga}_{x} \mathrm{Te}_{100-2 x}$ liquids performed at various temperatures. Black and red continues curves represent the scattering and simulation results, respectively. The calculated structure factor for pure tellurium is also represented [23], together with experimental results [32] at $973 \mathrm{~K}$. The numbers indicated on the left are the calculated Wright parameters.

which provides a good indication that the memory of the initial configurations has been lost. Subsequent simulations have been performed over typically $30-35$ ps for each target temperature $(913 \mathrm{~K}, 823 \mathrm{~K}, 773 \mathrm{~K}$, and $643 \mathrm{~K})$ and each composition. After equilibration at these temperatures, we have kept the last $25-30 \mathrm{ps}$ for the statistical analysis of structural, dynamic, and vibrational properties. The structural analysis concentrates essentially on the effect of composition on the $823 \mathrm{~K}$ isotherm (see below). For such temperatures, the calculated residual pressures were very small: $21.7 \mathrm{kPa}, 22.7 \mathrm{kPa}$, and $37.6 \mathrm{kPa}$ for the 14, 10, and $6 \%$ compositions, respectively. Figure 1 represents an example of an obtained structure for the case of $\mathrm{Ge}_{10} \mathrm{Ga}_{10} \mathrm{Te}_{80}$ at $823 \mathrm{~K}$.

\section{STRUCTURAL PROPERTIES}

For each composition $(6,10$, and $14 \%)$, the total experimental structure factor $S_{T}(k)$ is represented in Fig. 2, together with corresponding results from MD simulations performed at the corresponding temperatures. Similarly, we have compared the experimental and calculated total pair correlation functions $g(r)$ (Fig. 3).

\section{A. Qualitative analysis of the experimental data}

An interesting means used for the analysis of the structure builds on an order parameter defined by $S=S_{T}\left(k_{2}\right) / S_{T}\left(k_{1}\right)$ where $k_{1}$ and $k_{2}$ correspond to the positions of the two principal peaks of the total structure factor. As proposed in Refs. [33] and [34], this parameter turns out to be useful in order to distinguish between octahedral $(S<1)$ and tetrahedral $(S>1)$ local structure of liquids. In fact, tetrahedral liquids 


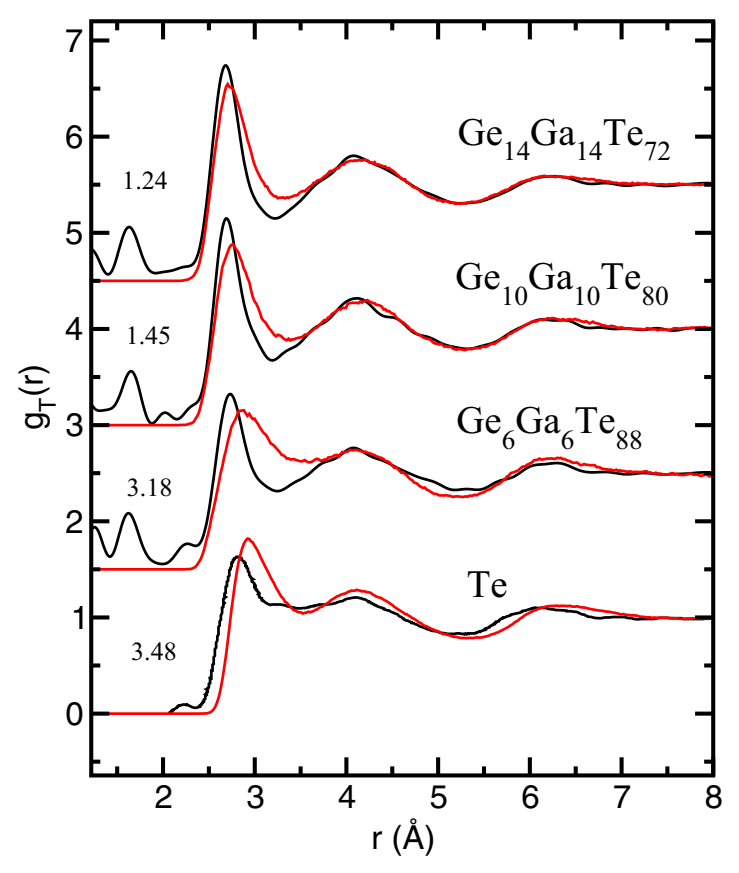

FIG. 3. Evolution of the total pair correlation function $g(r)$ in $\mathrm{Ge}_{x} \mathrm{Ga}_{x} \mathrm{Te}_{100-2 x}$ performed at various temperatures. Black and red curves represent the scattering and simulation results, respectively. The calculated $g(r)$ for pure tellurium is also represented [23], together with experimental results [32] at $973 \mathrm{~K}$. The numbers indicated on the left are the calculated Wright parameters.

such as silica [35] or $\mathrm{GeSe}_{2}$ [27] display a prominent secondary peak at $k=k_{2}$ that has a larger amplitude than the first peak at $k=k_{1}$. Conversely, octahedral liquids lead to $S<1$ as found in, e.g., liquid SnTe [33] or GeSbTe [23].

From the experimentally measured $S_{T}(k)$, we have estimated the values for $S$ which are given in Table I for the three considered liquids. It is seen that $S$ gradually increases with the addition of $\mathrm{Ge} / \mathrm{Ga}$ modifiers. While $\mathrm{Ge}_{10} \mathrm{Ga}_{10} \mathrm{Te}_{80}$ has a structure that is predominantly octahedral, the local order becomes more tetrahedral for $\mathrm{Ge}_{14} \mathrm{Ga}_{14} \mathrm{Te}_{72}$ and $S=1.06(1)$. This provides a first qualitative evidence that the addition of $\mathrm{Ge}$ and Ga modifies substantially the local structure and increases the tetrahedral character of the liquid.

It is instructive to compare the present results with previous estimations of the order parameter $S$ in Ge-Sb-Te melts [23]. For instance, at the same modifier composition $(x=6 \%)$, it was found $S=0.78$ for $\mathrm{Ge}_{6} \mathrm{Sb}_{6} \mathrm{Te}_{88}$ which indicates that there were more octahedra in the Sb-based structure than in the $\mathrm{Ga}$ counterpart $\left[S=0.90\right.$ (2) for $\mathrm{Ge}_{6} \mathrm{Ga}_{6} \mathrm{Te}_{88}$, Table I]. The $\mathrm{Sb} / \mathrm{Ga}$ substitution leads apparently to profound changes in the shortrange structure as also evidenced when both structure factors or pair correlation functions are being represented (Fig. 4). This feature is being recovered for the other compositions because one has $S=0.80$ in liquid $\mathrm{Ge}_{10} \mathrm{Sb}_{10} \mathrm{Te}_{80}$ [23] and $S=0.99$ (3) for the liquid $\mathrm{Ge}_{10} \mathrm{Ga}_{10} \mathrm{Te}_{80}$ (Table I). The situation is even more pronounced for the last composition (14\%) for which it has been found $S=1.06(1)$ and $S=0.81$ for $\mathrm{Ge}_{14} \mathrm{Ga}_{14} \mathrm{Te}_{72}$ and $\mathrm{Ge}_{14} \mathrm{Sb}_{14} \mathrm{Te}_{72}$, respectively. For this particular composition (14\%), the substitution leads to a strong increase of the tetrahedral character of the melts. A comparison with binary

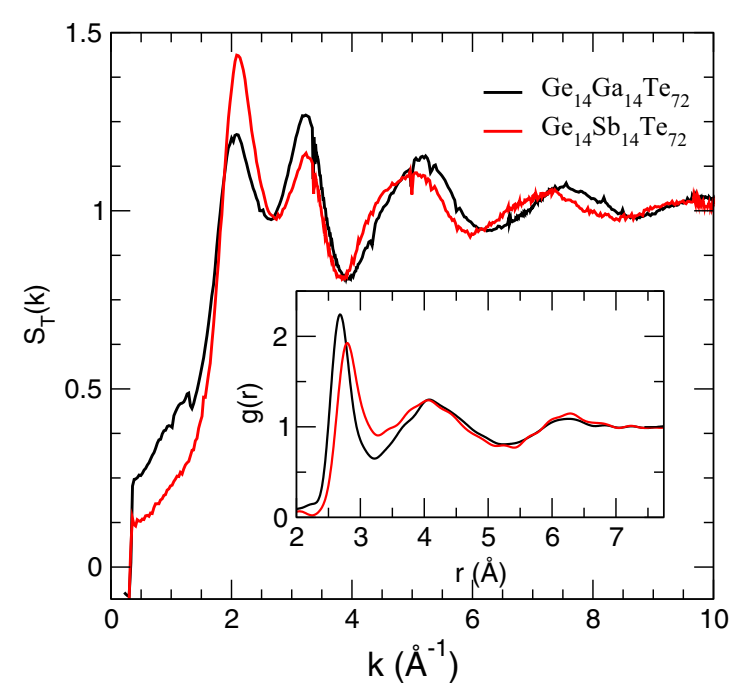

FIG. 4. Comparison between the experimental structure factors $S_{T}(k)$ and pair correlation functions $g(r)$ (inset) of liquid $\mathrm{Ge}_{14} \mathrm{Ga}_{14} \mathrm{Te}_{72}(873 \mathrm{~K})$ and $\mathrm{Ge}_{14} \mathrm{Sb}_{14} \mathrm{Te}_{72}$ (823 K) [23].

melts (Ge-Te) leads to similar conclusions. For instance, it has found [34] $S=0.83-0.86$ in liquid $\mathrm{Ge}_{20} \mathrm{Te}_{80}$ over the temperature range 700-923 K, and this value is much lower than the one obtained for the ternary system having the same amount of $\mathrm{Te}\left(\mathrm{Ge}_{10} \mathrm{Ga}_{10} \mathrm{Te}_{80}\right)$ for which $S \approx 1$ (Table I). We can therefore conclude that the substitution of germanium by gallium or the substitution of antimany by gallium increases $S$ and drives the liquid from an octahedral structure to a tetrahedral one.

The total measured and calculated pair correlation functions $g(r)$ are presented in Fig. 3 for all compositions of interest. We first note that the agreement evolves from poor to very good as the Ge/Ga composition $x$ is increased. The level of agreement can be quantified using the Wright parameter which evaluates a squared deviation between experimental and theoretical data [36]. The used functional for Te (PBEsol) is known to slightly overestimate the peak positions $\left(r_{1}^{\text {expt }}\right)$ in elemental Tellurium (2.91 $\AA$ [34] versus $2.82 \AA$ [25]) and this flaw can only be moderately cured using a more complex functional (Tao, Perdew, Staroverov, and Scuseria, TPSS [37]). Note that the use of TPSS burdens the computational cost and limits the possibility of extended investigations. As $x$ increases, the agreement automatically improves (as does the Wright parameter) because of a lesser contribution of the inappropriate Te functional to the structure, and for $\mathrm{Ge}_{14} \mathrm{Ga}_{14} \mathrm{Te}_{72}$ the simulated curve agrees now rather well with the experimental counterpart, the main features (peak amplitude and positions) being exactly reproduced (Table I), e.g., we find $r_{1}^{\text {expt }}=r_{1}^{\text {th }}=$ $2.70 \AA$ which corresponds to the first correlating distance of the liquid. The simulation leads, however, to a less structured liquid as detected from a larger minimum $g\left(r_{\min }\right)$ found. We furthermore note that with changing $x$ the distance to the first peak $\left(r_{1}^{\text {expt }}\right)$ decreases with $\mathrm{Ge} / \mathrm{Ga}$ content and evolves from $2.82 \AA$ for liquid Te to $2.70 \AA$, the trend being comparable to the one obtained from simulation $\left(r_{1}^{\mathrm{th}}\right)$. The second peak also evolves with decreasing Te content, and a reduction with $x$ is obtained (4.11 $\AA$ for $x=6 \%)$. This indicates a more compact 
structure for the second shell of neighbors at high $\mathrm{Ge} / \mathrm{Ga}$ content. The two main peak positions of the simulated $g(r)$ $\left(r_{1}^{\text {th }}\right.$ and $r_{2}^{\text {th }}$, see Table I) can also be compared to experimental findings. It is seen that while the first peak position (the bond distance at $r_{1}^{\text {th }}$ ) shows a difference with the experimental one $\left(r_{1}^{\text {expt }}\right)$, the agreement with experiments is very good for the secondary peak found at $r_{2}^{\text {th }} \simeq 4.11-4.22 \AA$. This tendency is true for all compositions, all also directly observed from Fig. 3 .

Using the total experimental pair correlation functions $g(r)$ and the experimentally estimated densities $\rho_{\text {expt }}$ (see Table I), the total coordination numbers:

$$
N_{\mathrm{expt}}^{\mathrm{tot}}=4 \pi \rho_{\mathrm{expt}} \int_{r_{0}}^{r_{\min }} r^{2} g(r) d r
$$

can be obtained as a function of Te content. $N_{\text {expt }}^{\text {tot }}$ values are listed in Table I, together with the value of the upper integration bound $r_{\min }$ representing the first minimum of $g(r)$. The lower integration bound $r_{0}$ is fixed to $2.20 \AA$ for all the mixtures. We can notice that the total coordination number does not change too much with increasing $\mathrm{Ge} / \mathrm{Ga}$ content given that it evolves between 3.21-3.48, the lower value found for $\mathrm{Ge}_{10} \mathrm{Ga}_{10} \mathrm{Te}_{80}$ $\left(N_{\text {expt }}^{\text {tot }}=3.21\right)$ being possibly linked with the smaller density $\left(0.0296 \AA^{-3}\right)$ of the liquid.

\section{B. Structural analysis using MD simulation}

\section{Comparison with experiments}

In Fig. 2, the experimental structure factors are compared with the total calculated structure factors $S_{T}(k)$ defined by:

$$
S_{T}(k)=\frac{\sum_{i, j} c_{i} c_{j} b_{i} b_{j} S_{i j}(k)}{\left(\sum_{i} c_{i} b_{i}\right)^{2}}
$$

and are extracted from the calculated partial structure factors $S_{i j}(k)$ by Fourier transforming the appropriate pair correlation functions. Here, the concentrations $c_{i}(i=\mathrm{Ge}, \mathrm{Ga}, \mathrm{Te})$ depend on the considered stoichiometry, and $b_{i}$ are the neutron scattering lengths given by $b_{\mathrm{Ge}}=8.185 \mathrm{fm}, \mathrm{b}_{\mathrm{Ga}}=7.288 \mathrm{fm}$, and $\mathrm{b}_{T e}=5.68 \mathrm{fm}$, respectively [38].

It can be remarked that the experimental data of $S_{T}(k)$ are very well reproduced over the entire range of wave vectors $k$, this statement being valid for all compositions, in contrast with the statement made above for the real space properties. When tracked with composition, we furthermore note that the agreement with the experimental function seems to increase with modifier composition, i.e., as one moves from $\mathrm{Ge}_{6} \mathrm{Ga}_{6} \mathrm{Te}_{86}$ to $\mathrm{Ge}_{14} \mathrm{Ga}_{14} \mathrm{Te}_{72}$. For instance, the calculated structure factor of $\mathrm{Ge}_{14} \mathrm{Ga}_{14} \mathrm{Te}_{72}$ (Fig. 2) shows the best resolved peaks with amplitudes and peak positions that match those measured from neutron scattering.

\section{Effect of composition}

The effect of composition can be analyzed from the evolution of both the total structure factor (Fig. 2) and the total radial distribution function (Fig. 3). Both first and second peaks in $g(r)$ are found to slightly vary with composition, e.g., the first peak shifts to shorter $r$ from $6 \%\left(r_{1}^{\text {th }}=2.86 \AA\right)$ to $14 \%\left(r_{2}^{\text {th }}=2.70 \AA\right.$ ) (see Table I). This result cannot be attributed only to the change in liquid density and is also
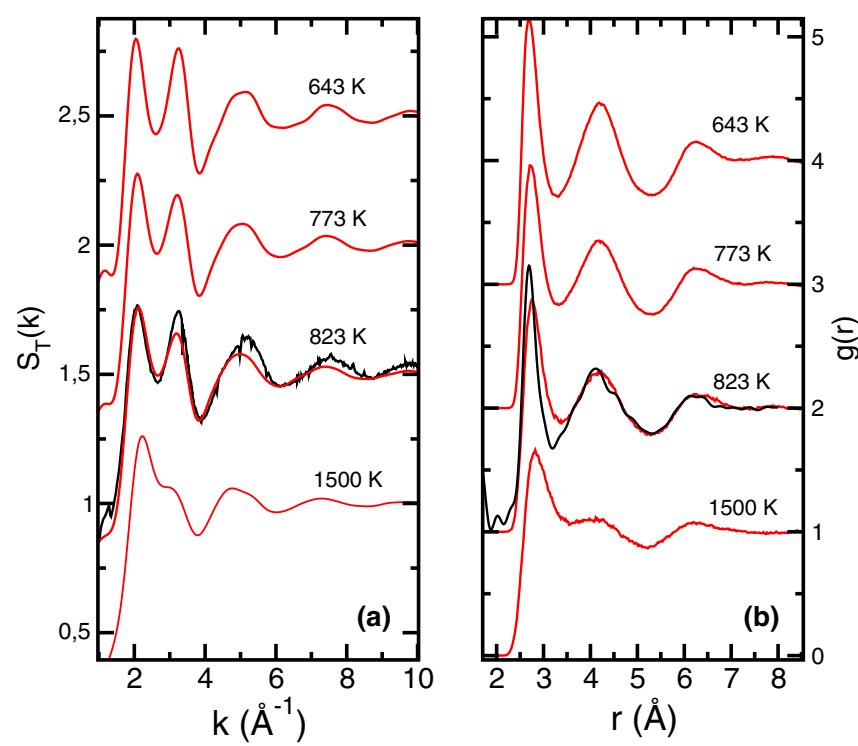

FIG. 5. (a) Total structure factor of $\mathrm{Ge}_{10} \mathrm{Ga}_{10} \mathrm{Te}_{80}$ liquid for different simulated temperatures. (b) Corresponding pair correlation function $g(r)$. The black curves are the experimental data from neutron scattering (same as Figs. 2 and 3).

linked to the occurrence of smaller long-range correlations as the concentration of $\mathrm{Ge}$ and $\mathrm{Ga}$ increases. A similar trend can be seen in the experimental first and second peaks of $g(r)$. In reciprocal space, the first peak of $S_{\mathrm{th}}(k)$ for compositions from $6 \%$ to $14 \%$ is found at the same position; in contrast, the second peak shows slight variations as composition change.

Similarly, the total coordination number $N_{\mathrm{th}}^{\text {tot }}$ calculated from the area of the first peak in the total radial distribution function (Fig. 3) using Eq. (1) leads to values that are close to those determined experimentally, and both total running coordination numbers $N_{\mathrm{th}}^{\mathrm{tot}}(r)$ and $N_{\mathrm{expt}}^{\mathrm{tot}}(r)$ are close for nearly all compositions. As previously emphasized [34], the differences emerging for the total coordination number thus only arise from the choice of the minimum $r_{\text {min }}$. For instance, we find $N_{\text {th }}^{\text {tot }}=3.67$ (at the minimum of $g(r), r_{\min }=3.37 \AA$ ) for $\mathrm{Ge}_{10} \mathrm{Ga}_{10} \mathrm{Te}_{80}$ that can be compared to the experimental results $\left(N_{\text {expt }}^{\text {tot }}=3.21\right.$ at $r_{\text {min }}=3.22 \AA$, see Table I).

\section{Effect of temperature}

In Fig. 5, we represent the calculated total structure factor $S_{T}(k)$ and pair correlation function $g(r)$ of $\mathrm{Ge}_{10} \mathrm{Ga}_{10} \mathrm{Te}_{80}$ for different simulated temperatures ranging from $1500 \mathrm{~K}$ down to $643 \mathrm{~K}$. In reciprocal space, one acknowledges a growth of the amplitude $S\left(k_{1}\right)$ of the principal peak which is, according to the definition of the order parameter, an indication that the liquid becomes more tetrahedral as the temperature is decreased. Note that this temperature evolution leads to the usual sharpening of the main diffraction peaks (i.e., at $k_{1}$ and $k_{2}$ which turn out to be nearly temperature independent) as usually observed in supercooled liquids.

In real space, the high temperature liquid $(1500 \mathrm{~K})$ does not exhibit any structuration and the second peak arising from a second shell of neighbors is barely visible. With decreasing temperature, the first correlation distance at $r_{1}^{\text {th }}$ is found to 


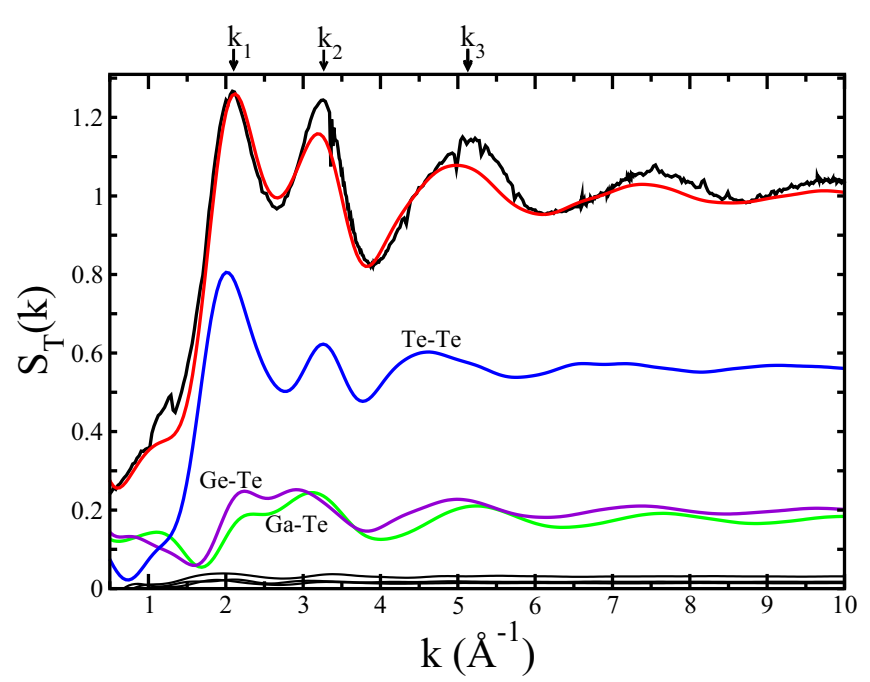

FIG. 6. A typical decomposition of the total simulated structure factor $S_{T}(k)$ of liquid $\left(823 \mathrm{~K}\right.$ ) $\mathrm{Ge}_{10} \mathrm{Ga}_{10} \mathrm{Te}_{80}$ (same as Fig. 2) into partial structure factor $\left(2-\delta_{i j}\right) c_{i} c_{j} b_{i} b_{j} S_{i j}(k) /\langle b\rangle^{2}$. Blue, violet, and green curves represent $S_{\mathrm{TeTe}}(k), S_{G e T e}(k)$, and $S_{\mathrm{GaTe}}(k)$, respectively. The position of the main peaks $\left(k_{1}, k_{2}, k_{3}\right)$ are indicated on the top axis.

decrease and evolves from $2.84 \AA$ at $1500 \mathrm{~K}$ down to $2.76 \AA$ for $823 \mathrm{~K}$ (Table I) and $773 \mathrm{~K}$, and finally $2.72 \AA$ for $643 \mathrm{~K}$. Similar results and global tendencies are obtained for the other ternary compositions.

\section{Partial contributions}

\section{Reciprocal space}

For all compositions, the total structure factors $S_{T}(k)$ are essentially dominated by three pairs: $\mathrm{Ge}-\mathrm{Te}, \mathrm{Ga}-\mathrm{Te}$, and $\mathrm{Te}-$ Te. Figure 6 shows, as an example, the contributions of such pairs to the simulated $S_{T}(k)$ in the $\mathrm{Ge}_{10} \mathrm{Ga}_{10} \mathrm{Te}_{80}$ liquid. All other pairs are found to contribute to less than $\simeq 3 \%$, given the chosen interval in composition $(0 \leqslant x \leqslant 14 \%)$, and their neutron coherent lengths $b_{i}$ [see Eq. (2)].

From Fig. 6, one furthermore detects which pair contributes to the observed peaks of the total measured or simulated structure factor (Fig. 2). It is found that $S_{\mathrm{TeTe}}(k)$ contributes to all relevant peaks, i.e., first and second peak at $k_{1} \approx 2.0 \AA^{-1}$ and $k_{2} \approx 3.2 \AA^{-1}$, but also to the third broad peak found at $k_{3} \approx 4.6 \AA^{-1}$. On the other hand, the two other relevant partials, Ge-Te and Ga-Te, have mostly contributions at $k_{1}$ and $k_{2}$.

Such partial structure factors $S_{i j}(k)$ can be studied as a function of composition (Figs. 7 and 8), and one can remark that the most important changes are found in the Te-Te partial which has a growing secondary principal peak at $k=k_{2}$ with increasing Te content, together with an increase of the amplitude of the principal peak at $k=k_{1}$. The latter feature is a qualitative indication that the decrease of the number of Te atoms leads to emerging correlations that build up at larger distances (i.e., lower wave vector $k$ ).

Both partials Ge-Te and Ga-Te do not have at all the same shape because $S_{\mathrm{GaTe}}(k)$ has a more intense peak at $k=k_{2}$ and

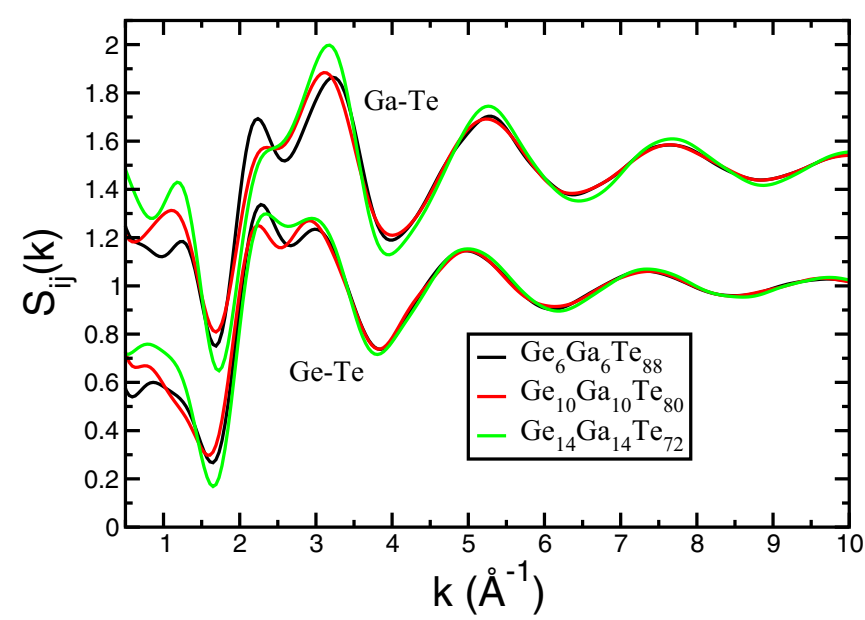

FIG. 7. Evolution of the simulated partial structure factor $S_{\mathrm{GeTe}}(k)$ and $S_{\mathrm{GaTe}}(k)$ with composition $x$ in $\mathrm{Ge}_{x} \mathrm{Ga}_{x} \mathrm{Te}_{100-2 x}$ at $823 \mathrm{~K}$.

a strong contribution to the total $S_{T}(k)$. On the other hand, $S_{\mathrm{GeTe}}$ displays two broad peaks with similar intensities located at $k_{1}$ and $k_{2}$. One can, thus, argue that structural correlations induced by $\mathrm{Ge}$ or $\mathrm{Ga}$ cross links are not equivalent and affect structural correlations at $k<4 \AA^{-1}$ in a different way. From the peak evolution with $x$, we can also argue that the evolution of the order parameter $S$ and the principal peak at $k=k_{1}$ is driven by the decrease of Ga-Te correlations that cannot be counterbalanced by the growth of the principal peak in $S_{\mathrm{TeTe}}(k)$ (Fig. 8).

Both Ga-Te and Ge-Te partial structure factors are found to display increased oscillations at $2 \AA^{-1} \leqslant k \leqslant 4 \AA^{-1}$. For instance, the amplitude of the second shoulder peak found at $k \simeq 2.1 \AA^{-1}$ in the function $S_{\mathrm{GaTe}}(k)$ is found to decrease substantially between the $6 \%$ and $10 \%$ liquids, and leads to a separate peak at low Ge/Ga content (see Fig. 2). Similarly, we find that the two first peaks of $S_{\mathrm{GeTe}}(k)$ nearly merge for $\mathrm{Ge}_{14} \mathrm{Ga}_{14} \mathrm{Te}_{72}$ and this contributes to a broadening of the principal peak of the total structure factor (see Fig. 2).

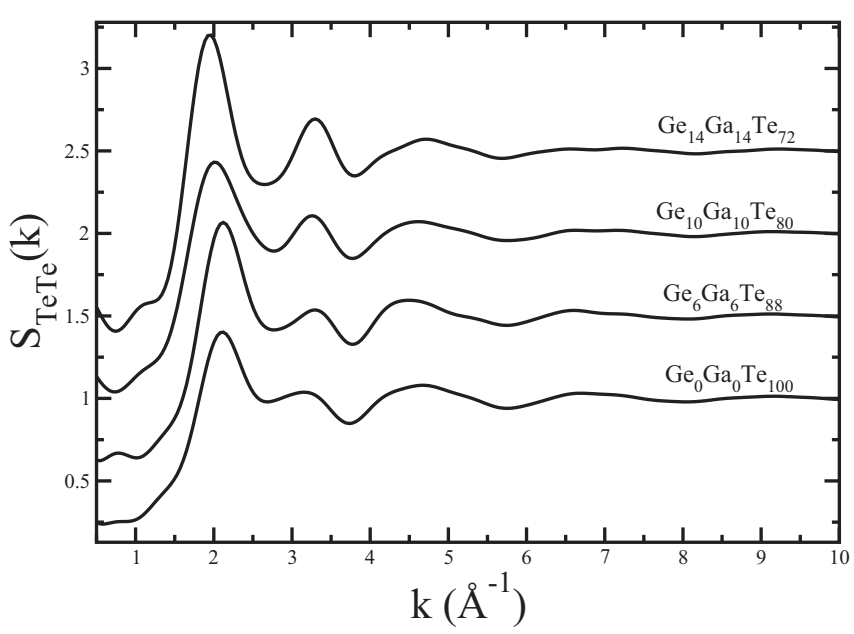

FIG. 8. Evolution of the simulated partial structure factor $S_{\mathrm{TeTe}}(k)$ with composition $x$ in $\mathrm{Ge}_{x} \mathrm{Ga}_{x} \mathrm{Te}_{100-2 x}$ at $823 \mathrm{~K}$. 

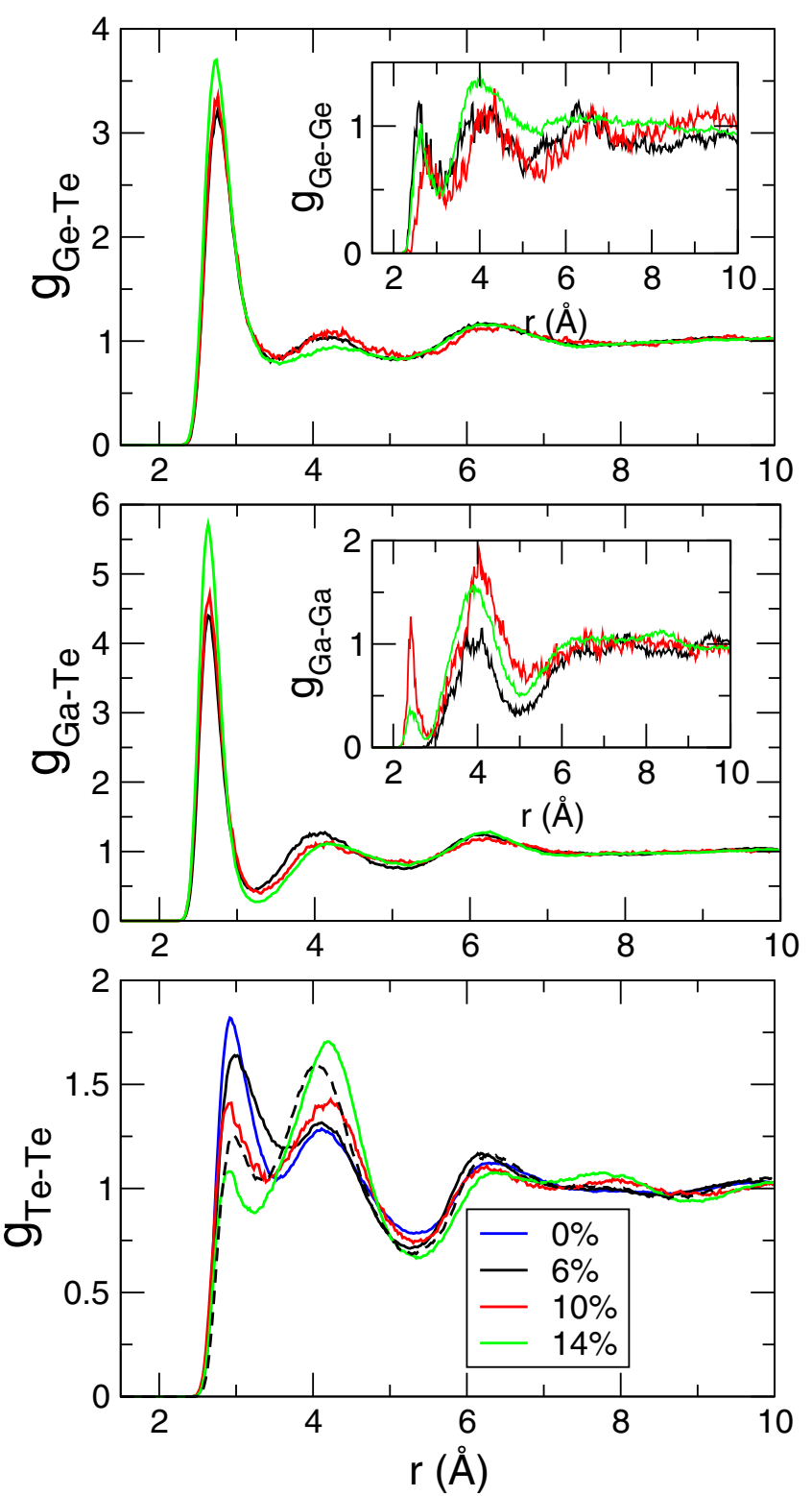

FIG. 9. Evolution of the simulated partial pair correlation functions $\mathrm{g}_{i j}(\mathrm{r})$ at fixed temperature $(823 \mathrm{~K})$ as a function of composition $x$ in $\mathrm{Ge}_{x} \mathrm{Ga}_{x} \mathrm{Te}_{100-2 x}$ liquids. The broken black curve in the panel $g_{\mathrm{TeTe}}$ represents the Te-Te pair correlation function of $\mathrm{Ge}_{10} \mathrm{Sb}_{10} \mathrm{Te}_{80}$.

\section{Real space properties at $823 \mathrm{~K}$}

The analysis of the structure in real space can be characterized from the partial radial distribution functions, as shown in Fig. 9. A certain number of features should be emphasized. First, we note that Ge-Ge and Ga-Ga homopolar bonds exist for all compositions, including for the one with the highest Te content $\mathrm{Ge}_{6} \mathrm{Ga}_{6} \mathrm{Te}_{88}$ (Fig. 9, insets), the former leading to a peak in the partial pair correlation function $g_{\mathrm{GeGe}}(r)$ at $r \approx 2.61 \AA$, while the latter exhibits a peak at $r \approx 2.41 \AA$ in $g_{\mathrm{GaGa}}(r)$ at $10 \%$ and $14 \%$, meanwhile at $6 \%$ the first peak is at $r \approx 3.7 \AA$. Furthermore, it is seen that the fraction of Ge-Ge and Ga-Ga bonds is negligible compared to the other bond populations (Ge-Te, Te-Te, and $\mathrm{Ga}-\mathrm{Te}$ ) which dominate the structure in the Te-rich liquids, the fraction of $\mathrm{Ge}-\mathrm{Ge}$ and $\mathrm{Ga}-$
TABLE II. Cutoff distance $r_{i j}^{m}$ [minimum of the corresponding function $\left.g_{i j}(r)\right]$, and calculated partial coordination numbers $n_{i j}$ with $i, j=(\mathrm{Ge}, \mathrm{Ga}, \mathrm{Te})$ at different compositions $x$ at $823 \mathrm{~K}$ in $\mathrm{Ge}_{x} \mathrm{Ga}_{x} \mathrm{Te}_{100-2 x}$ liquids. Results for the $\mathrm{Ge}_{14} \mathrm{Ga}_{14} \mathrm{Te}_{72}$ liquid are compared to the parent $\mathrm{Ge}_{14} \mathrm{Sb}_{14} \mathrm{Te}_{72}$ [23].

\begin{tabular}{llcccccc}
\hline \hline$x$ & & $\mathrm{GeGe}$ & $\mathrm{GeGa}$ & $\mathrm{GeTe}$ & $\mathrm{GaGa}$ & $\mathrm{GaTe}$ & $\mathrm{TeTe}$ \\
\hline 6 & $r_{i j}^{m}(\AA)$ & 3.03 & 2.77 & 3.48 & 4.98 & 3.21 & 3.63 \\
& $n_{i j}$ & 0.06 & 0.01 & 4.76 & 0.44 & 3.71 & 4.25 \\
10 & $r_{i j}^{m}(\AA)$ & 3.23 & 2.96 & 3.54 & 2.85 & 3.30 & 3.41 \\
& $n_{i j}$ & 0.1 & 0.02 & 4.48 & 0.06 & 3.78 & 2.42 \\
\multirow{2}{*}{14} & $r_{i j}^{m}(\AA)$ & 3.03 & 2.85 & 3.59 & 2.78 & 3.26 & 3.26 \\
& $n_{i j}$ & 0.16 & 0.02 & 4.30 & 0.04 & 3.43 & 1.40 \\
& & $\mathrm{GeGe}$ & $\mathrm{GeSb}$ & $\mathrm{GeTe}$ & $\mathrm{SbSb}$ & $\mathrm{SbTe}$ & $\mathrm{TeTe}$ \\
\multirow{2}{*}{$14[23]$} & $n_{i j}$ & 0.45 & 0.27 & 3.75 & 0.44 & 4.53 & 1.62 \\
\hline \hline
\end{tabular}

Ga starting to increase only for $x>10 \%$, a feature also noticed in a similar study on amorphous Ge-Te [39]. Specifically, we find at the minimum $r_{m}$ of the pair correlation function a fraction of $\mathrm{Ge}-\mathrm{Ge}$ bonds that evolves in a nonmonotonous fashion with composition, i.e., it is found $15.1 \%$ and $15.9 \%$ for $\mathrm{Ge}_{14} \mathrm{Ga}_{14} \mathrm{Te}_{72}$ and $\mathrm{Ge}_{6} \mathrm{Ga}_{6} \mathrm{Te}_{88}$, respectively, whereas one has $3.4 \% \mathrm{Ge}-\mathrm{Ge}$ bonds only for $\mathrm{Ge}_{10} \mathrm{Ga}_{10} \mathrm{Te}_{80}$. This anomaly is detected from the lower amplitude of the first peak of the partial $g_{\mathrm{GeGe}}$. The fraction of homopolar Ga-Ga is found to be much smaller (1\%) except for $\mathrm{Ge}_{10} \mathrm{Ga}_{10} \mathrm{Te}_{80}$ which has a fraction of $2.1 \%$ as also highlighted in the amplitude of the corresponding Ga-Ga partial pair correlation function (Fig. 9). With increasing $\mathrm{Ge} / \mathrm{Ga}$ content, such homopolar distances tend to remain constant, for instance we find $d_{\mathrm{GeGe}} \approx 2.61 \AA$ for all liquids. An inspection of the Te-Te partials indicates that the number of bond distances typical of chain fragments ( $3 \AA$ [25]) decrease with growing $x$ so that the typical correlating distance at large $\mathrm{Ge} / \mathrm{Ga}$ content is found at $\simeq 4 \AA$, a distance that corresponds to the interchain distance in amorphous Tellurium [25].

\section{Coordination numbers}

In Table II, we report the partial pair coordinations $n_{i j}$ calculated from the partial pair correlation functions (Fig. 9). With increasing $\mathrm{Ge} / \mathrm{Ga}$ content, the partial coordination numbers $n_{\mathrm{GeTe}}$ decrease. This decrease is related to the growth of homopolar coordination number $n_{\mathrm{GeGe}}$, and a similar trend with $x$ is obtained for $n_{\mathrm{GaGa}}$.

Corresponding total coordination numbers can then be calculated (Table III) using

$$
n_{i}=n_{i i}+\sum_{i \neq j} n_{i j}
$$

and, e.g., $(100-2 x) n_{\mathrm{TeGe}}=x n_{\mathrm{GeTe}}$. Results show that the atoms do not follow the 8-N rule ( $\mathrm{N}$ being the number of $\mathrm{p}$ and s electrons). One finds, indeed, that $\mathrm{Ge}$ atoms have a coordination number of about 4.48-4.83, whereas Ga atoms have a slightly lower coordination number $\left(n_{\mathrm{Ga}}=3.49-4.26\right)$. This arises from the fact that the dominant geometry of $\mathrm{Ga}$ is tetrahedral, whereas Ge atoms can be found in two local geometries, defect octahedral and tetrahedral [40]. Te atoms 
TABLE III. Calculated coordination numbers $n_{i}$ at different compositions $x$ in the $(823 \mathrm{~K}) \mathrm{Ge}_{x} \mathrm{Ga}_{x} \mathrm{Te}_{100-2 x}$ liquids.

\begin{tabular}{lcccc}
\hline \hline System & & $\mathrm{Ge}$ & $\mathrm{Ga}$ & $\mathrm{Te}$ \\
\hline $\mathrm{Ge}_{6} \mathrm{Ga}_{6} \mathrm{Te}_{88}$ & $n_{i}$ & $4.83 \pm 0.05$ & $4.26 \pm 0.10$ & $4.82 \pm 0.09$ \\
$\mathrm{Ge}_{10} \mathrm{Ga}_{10} \mathrm{Te}_{80}$ & $n_{i}$ & $4.60 \pm 0.05$ & $3.86 \pm 0.10$ & $3.45 \pm 0.09$ \\
$\mathrm{Ge}_{14} \mathrm{Ga}_{14} \mathrm{Te}_{72}$ & $n_{i}$ & $4.48 \pm 0.05$ & $3.49 \pm 0.10$ & $2.90 \pm 0.09$ \\
\hline \hline
\end{tabular}

have a coordination number that decreases as $x$ increases, i.e., one finds $n_{T e}=2.90-4.82$ for the different compositions.

An inspection of the detail of the coordination numbers (Table IV) provides further insight into the network topology and its change with composition. Here, one follows the coordination distributions of $\mathrm{Ge}^{l}, \mathrm{Ga}^{l}$, and $\mathrm{Te}^{l}(l=\mathrm{I}, \mathrm{II}, \mathrm{III}, \mathrm{IV}, \mathrm{V}, \mathrm{VI})$. In the investigated liquids, the dominant coordination of the cross-linking elements is $\mathrm{Ge}^{\mathrm{IV}}$ and $\mathrm{Ga}^{\mathrm{IV}}$, the latter being dominant for all compositions (77.2-82.8\%) with a minority of defect coordinations. Conversely, Ge displays a broader distribution of coordinated species with a majority of $\mathrm{Ge}^{\mathrm{IV}}$ and substantial amounts of defect coordinations $\mathrm{Ge}^{\mathrm{III}}$ and $\mathrm{Ge}^{\mathrm{V}}$. Here, $\mathrm{Ge}^{\mathrm{v}}$ seems to be mostly present in the chalcogen-rich domain (36.8\% for $\mathrm{Ge}_{6} \mathrm{Ga}_{6} \mathrm{Te}_{84}$ ) and decreases to about $10 \%$ for $\mathrm{Ge}_{14} \mathrm{Ga}_{14} \mathrm{Te}_{72}$ (13.6\%). The opposite trend is found for $\mathrm{Ge}^{\mathrm{III}}$ which increases with $\mathrm{Ge} / \mathrm{Ga}$ content from $14.7 \%$ to $30.1 \%$.Tellurium is found in I, II, and III coordinated environments and the fraction of terminal $\mathrm{Te}^{\mathrm{I}}$ increases with the cross-link density $(x)$.

\section{E. Bond angles and fraction of tetrahedra}

Figure 10 displays the most important bond angle distributions (BAD) present in the liquid structures for the different compositions. It is found that the effect of composition is barely observed on such bond angle distributions and the most important ones are those involved in heteropolar bondings such as Te-Ge-Te and Te-Ga-Te which show almost no change over $6 \%$ and $10 \%$, whereas a slight change is obtained for the $\mathrm{Ge}_{14} \mathrm{Ga}_{14} \mathrm{Te}_{72}$ composition with an increased amplitude for the main angular contribution. For Te-Ge-Te [Fig. 10(b)], this contribution is located at around $95^{\circ}$ identified with a defective octahedral geometry for the germanium atoms, given that one also obtains a tail at $165^{\circ}$. Such features have been found in

TABLE IV. Calculated coordination distribution in liquid (823 K) $\mathrm{Ge}_{x} \mathrm{Ga}_{x} \mathrm{Te}_{100-x}$. The cutoff has been taken at each respective minimum of the pair distribution function.

\begin{tabular}{|c|c|c|c|c|c|c|}
\hline Composition & & I & II & III & IV & $\mathrm{V}$ \\
\hline \multirow[t]{3}{*}{$\mathrm{Ge}_{6} \mathrm{Ga}_{6} \mathrm{Te}_{88}$} & $\mathrm{Ge}$ & & 0.5 & 14.7 & 48.0 & 36.8 \\
\hline & $\mathrm{Ga}$ & & & 5.1 & 79.2 & 15.7 \\
\hline & $\mathrm{Te}$ & 12.0 & 35.9 & 35.8 & 14.1 & 2.1 \\
\hline \multirow[t]{3}{*}{$\mathrm{Ge}_{10} \mathrm{Ga}_{10} \mathrm{Te}_{80}$} & $\mathrm{Ge}$ & & 3.4 & 28.8 & 55.3 & 12.9 \\
\hline & $\mathrm{Ga}$ & & 0.8 & 10.3 & 77.2 & 11.7 \\
\hline & $\mathrm{Te}$ & 24.2 & 44.3 & 26.0 & 5.1 & 0.4 \\
\hline \multirow[t]{3}{*}{$\mathrm{Ge}_{14} \mathrm{Ga}_{14} \mathrm{Te}_{72}$} & $\mathrm{Ge}$ & & 3.1 & 30.1 & 53.6 & 13.6 \\
\hline & $\mathrm{Ga}$ & & 0.4 & 7.1 & 82.8 & 9.8 \\
\hline & $\mathrm{Te}$ & 30.5 & 42.7 & 22.3 & 4.3 & 0.3 \\
\hline
\end{tabular}

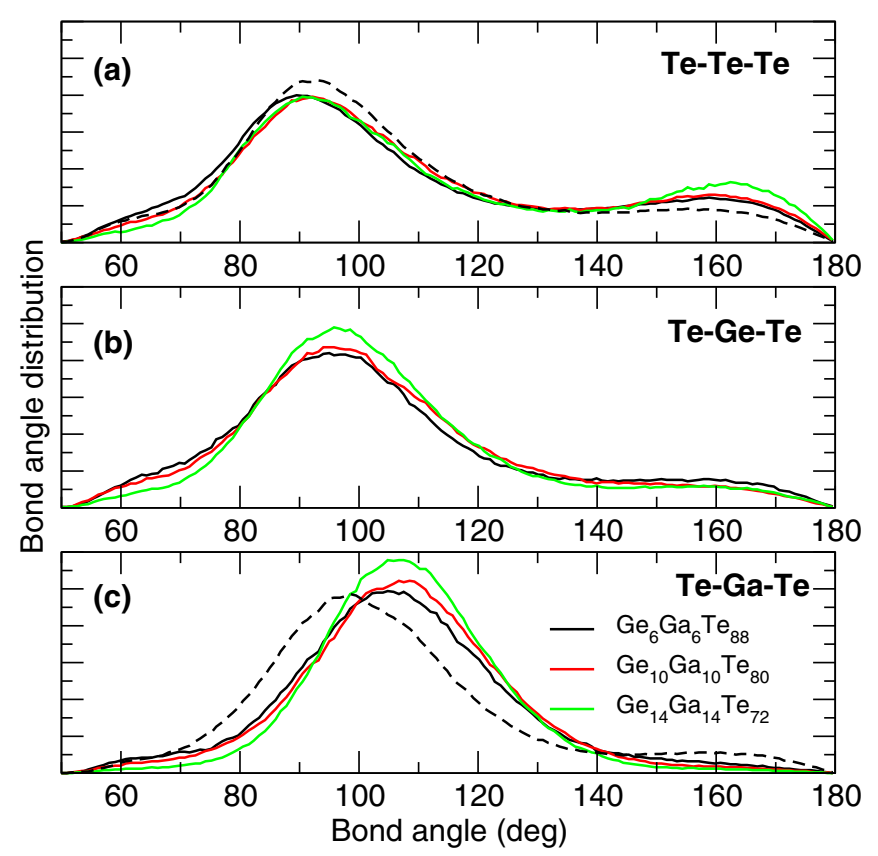

FIG. 10. Evolution of the simulated bond angle distributions Te$\mathrm{Te}-\mathrm{Te}$ (a), Te-Ge-Te (b), and Te-Ga-Te (c) at fixed temperature (823 K) as a function of composition $x$ in $\mathrm{Ge}_{x} \mathrm{Ga}_{x} \mathrm{Te}_{100-2 x}$ liquids. In panel (a), the broken curve represents the Te-Te-Te bond angle distribution of liquid Te [34]. In panel (c), the broken curve corresponds to the Te-Sb-Te BAD [23] and highlights the octahedral character of the bonding. A cutoff of $3.2 \AA$ has been used.

the amorphous phase [10] for $\mathrm{Ge}_{11} \mathrm{Ga}_{11} \mathrm{Te}_{78}$. The situation contrasts with the one obtained for Te-Ga-Te which exhibits a strong peak centered at $109^{\circ}$ and indicates that $\mathrm{Ga}$ is mostly in tetrahedral environment as also determined for the amorphous phase from different experimental techniques and DFT based simulations $[10,11]$. The change from $\mathrm{Sb}$ to $\mathrm{Ga}$ atoms (i.e., comparing a GST [23] and a GGT liquid) do not change the conclusions concerning the $\mathrm{Ge}$ based angular distributions, although a more sharp main peak is acknowledged for Te-GeTe BADs [black broken curve in Fig. 11(a)]. Conversely the BADs associated with the other element swith, indeed, from an octahedral environment (for Sb in GST) to a tetrahedral one (for Ga in GGT).

The presence of homopolar bonds does not alter this global picture albeit other angular correlations emerge as revealed once Te-Ge-Te and Te-Ge-Ge are being compared [Fig. 11(a)], or Te-Ga-Te and Te-Ga-Ga. [Fig. 11(b)]. The former indicates that the presence of homopolar Ge-Ge bonds leads to a growing fraction of tetrahedra as detected from the shift of the BAD maximum to larger angles, an indication that more geometries with tetrahedral angles $\left(109^{\circ}\right)$ are present in the liquid structure. The presence of a more intense peak at $\simeq 55^{\circ}$ is the manifestation of the presence of edge-sharing local geometries which involves a typical Ge-Ge correlating distance that can have a particular signature in neutron scattering studies [41] and a typical sharp angle. This contribution is enhanced for the $\mathrm{Ge}_{10} \mathrm{Ga}_{10} \mathrm{Te}_{80}$ liquid (not shown). Similarly, it is seen that the presence of homopolar Ga-Ga bonds also leads to the presence of a sharp peak at about $\simeq 60^{\circ}$ which reveals triangular 


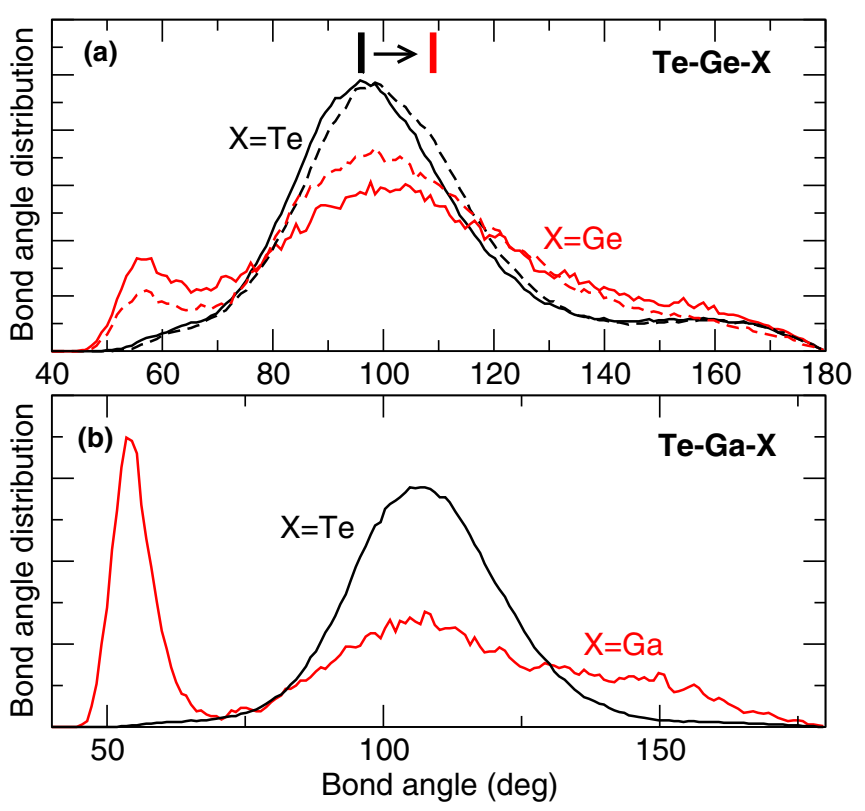

FIG. 11. Effect of homopolar bonds on the bond angle distributions Te-Ge-X (a) and Te-Ga-X (b) in $\mathrm{Ge}_{14} \mathrm{Ga}_{14} \mathrm{Te}_{72}$ liquids $(823 \mathrm{~K}$ ). (a) $\mathrm{X}=\mathrm{Te}$ [same as Fig. 10(b)] or $\mathrm{X}=\mathrm{Ge}$ (red curve). (b) $\mathrm{X}=\mathrm{Te}$ [same as Fig. $10(\mathrm{c})]$ or $\mathrm{X}=\mathrm{Ga}$ (red curve). In panel (a) are also represented corresponding results for $\mathrm{Ge}_{14} \mathrm{Sb}_{14} \mathrm{Te}_{72}$ [23] (broken curves). The arrow marks the shift of the average angle due to the presence of Ge-Ge homopolar bonds.

geometries but no modification of the tetrahedral character is acknowledged.

We furthermore note that the Te-Te-Te bond angle distribution is found to be extremely close to the one calculated for elemental Te $[x=0 \%$, broken curve in Fig. 10(a)]. This indicates that the angles involved in such Te-Te-Te fragments are weakly affected by the $\mathrm{Ge} / \mathrm{Ga}$ induced crosslinking of the structure. A visual inspection of the structure finally indicates that Te-Te-Ge and Te-Te-Ga BADs (not shown) have an additional contribution at $\simeq 60^{\circ}$ (with respect to Te-Te-Te) that we identify with angles defined by three atoms found within the equatorial plane of Te-centered defective octahedra.

While the global analysis (Fig. 10) remains at a qualitative level, we have used a recently introduced method $[40,42]$ to determine exactly without any ambiguity the fraction of $\mathrm{Ge}$ and Ga tetrahedra in the structure. It is based on the enumeration of angular topological constraints [43] that permits to estimate angular excursions. These are computed on-the-fly from the MD trajectory, the number of such angular constraints serving as a parameter for the identification of a tetrahedral geometry. The latter is, indeed, defined by six rigid angles [43] that give rise to corresponding low angular standard deviations. When such an analysis is performed on the Ge-Ga-Te liquids, one obtains a fraction $\eta_{T}$ of Ge tetrahedra that is always lower than for Ga as already qualitatively detected from Fig. 10 . We find for instance that $\eta_{T}=16.6 \%$ and $77.7 \%$ for $\mathrm{Ge}$ and $\mathrm{Ga}$ in $\mathrm{Ge}_{6} \mathrm{Ga}_{6} \mathrm{Te}_{88}$, respectively (Table V). The effect of composition seems to indicate that the addition of cross links increases the tetrahedral character of Ge because $\eta_{T}$ increases from about $10-15 \%$ to nearly $25 \%$ for the $\mathrm{Ge}_{14} \mathrm{Ga}_{14} \mathrm{Te}_{72}$ composition. Conversely, Ga atoms are not affected by the
TABLE V. Calculated fraction (in \%) of tetrahedra $\eta_{T}$ for Ge and $\mathrm{Ga}$ atoms using topological angular constraints (see methods in Ref. [40]). Cutoffs of $3.2 \AA$ and $18^{\circ}$ have been used. A comparison with a $\mathrm{Ge}_{20} \mathrm{Te}_{80}$ liquid (825 $\mathrm{K}$ ) is made [34].

\begin{tabular}{lcc}
\hline \hline System & $\mathrm{Ge}$ & $\mathrm{Ga}$ \\
\hline $\mathrm{Ge}_{6} \mathrm{Ga}_{6} \mathrm{Te}_{88}$ & $16.6 \pm 3.3$ & $77.7 \pm 5.5$ \\
$\mathrm{Ge}_{10} \mathrm{Ga}_{10} \mathrm{Te}_{80}$ & $10.0 \pm 3.3$ & $73.3 \pm 3.3$ \\
$\mathrm{Ge}_{14} \mathrm{Ga}_{14} \mathrm{Te}_{72}$ & $23.8 \pm 4.8$ & $73.8 \pm 4.8$ \\
$\mathrm{Ge}_{20} \mathrm{Te}_{80}[34]$ & $45.0 \pm 2.5$ & \\
$\mathrm{a}-\mathrm{Ge}_{10} \mathrm{Si}_{10} \mathrm{Te}_{80}[42]$ & 24.6 & \\
\hline \hline
\end{tabular}

change in structure and remain in predominant tetrahedral character for all compositions with nearly the same fraction $\left(\eta_{T} \simeq 75 \%\right)$.

The addition of $\mathrm{Ga}$ atoms into $\mathrm{Ge}-\mathrm{Te}$ based liquids seem to decrease the overall tetrahedral character of $\mathrm{Ge}$ atoms, an effect that appears to be somewhat more pronounced when compared to a similar telluride (amorphous $\mathrm{Ge}_{10} \mathrm{Si}_{10} \mathrm{Te}_{80}$ ) for which the fraction of Ge tetrahedra was found [42] to be of about 25\% (Table V). For the same amount of Te, a comparison of the binary liquid $\mathrm{Ge}_{20} \mathrm{Te}_{80}$ and the ternary $\mathrm{Ge}_{10} \mathrm{Ga}_{10} \mathrm{Te}_{80}$ furthermore shows that $\eta_{T}$ reduces from about $45 \%$ to about $10.0 \%$ (Table V), respectively. Note that the fraction found for liquid $\mathrm{Ge}_{20} \mathrm{Te}_{80}$ is compatible with the one obtained from MD simulations in the amorphous phase (54.6\%) and also consistent with measurements from Mössbauer spectroscopy (41.6\% for $\mathrm{Ge}_{18} \mathrm{Te}_{82}$ ) [40].

\section{VIBRATIONAL PROPERTIES}

The measured vibrational density of states (VDOS) $g(\omega)$ of the $\mathrm{Ge}_{x} \mathrm{Ga}_{x} \mathrm{Te}_{100-2 x}$ liquids are presented in Fig. 12, and the calculated $g(\omega)$ is obtained using the Fourier transform of the

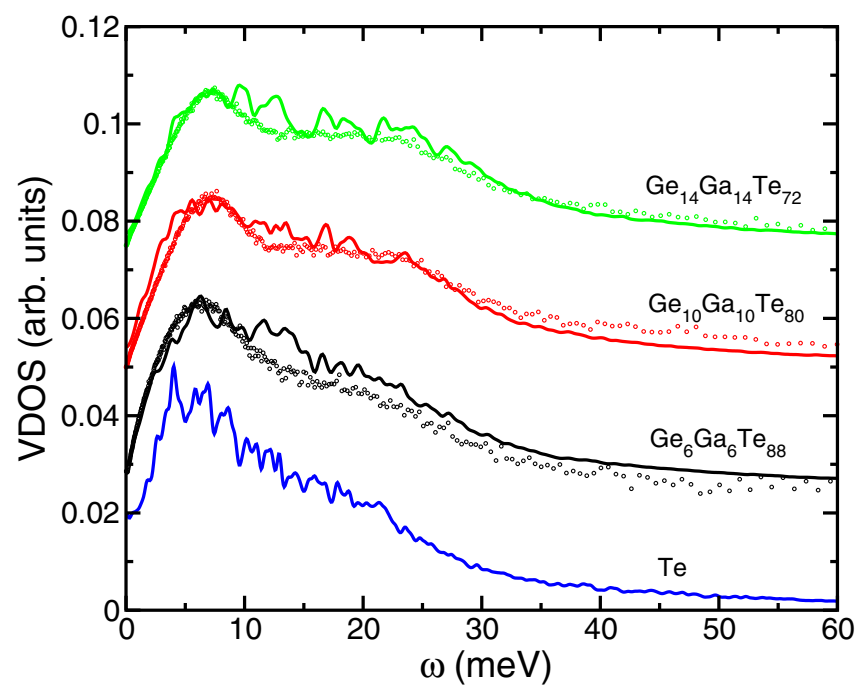

FIG. 12. Total measured (dots) and calculated (curves) vibrational density of states (VDOS) in $\mathrm{Ge}_{x} \mathrm{Ga}_{x} \mathrm{Te}_{100-2 x}$ liquids. The VDOS of liquid Te has been added for comparison [23]. 


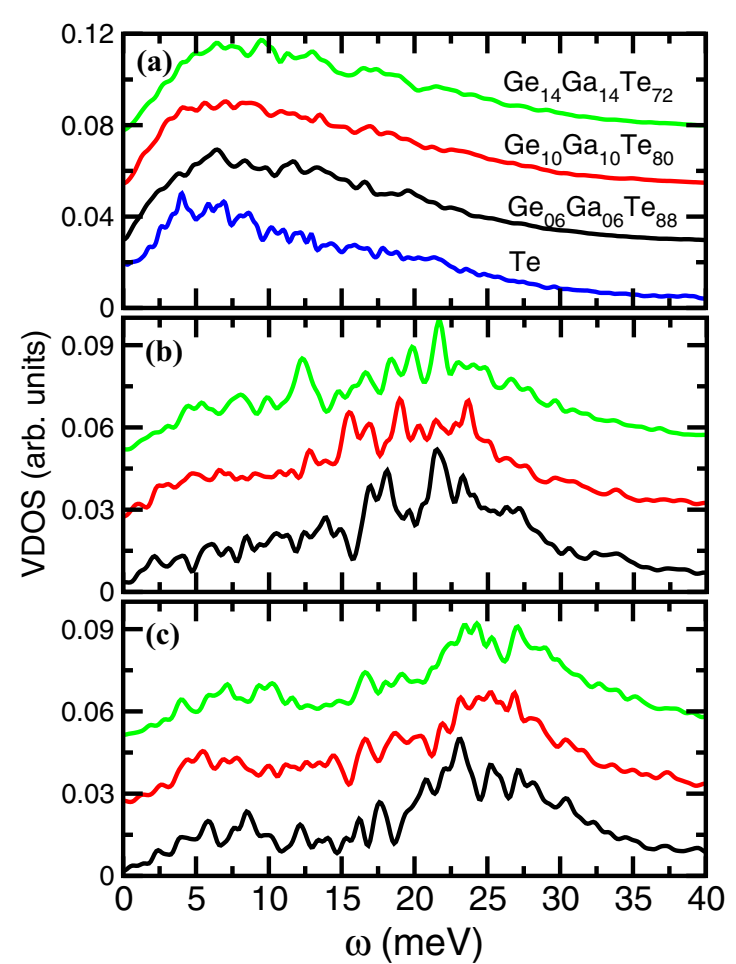

FIG. 13. Calculated partial vibrational density of states (VDOS) for different $\mathrm{Ge}-\mathrm{Ga}-\mathrm{Te}$ liquids at fixed temperature $T \simeq 823 \mathrm{~K}$. Contributions from $\mathrm{Te}(\mathrm{a}), \mathrm{Ge}(\mathrm{b})$, and $\mathrm{Ga}$ (c) atoms.

velocity-velocity autocorrelation function:

$$
g(\omega)=\frac{1}{N k_{B} T} \sum_{j=1}^{N} \int_{-\infty}^{\infty}\left\langle\mathbf{v}_{j}(t) \cdot \mathbf{v}_{j}(0)\right\rangle e^{i \omega t} d t .
$$

We first remark that the MD simulations lead to a rather good agreement with the measured VDOS. As seen from Fig. 12, a large part of the spectra is very well reproduced and this statement is true for all considered compositions. Similarly to previous studies of other liquid tellurides [23,34], we find that the calculated VDOS deviates from the experimental spectra for $\omega \geqslant 40 \mathrm{meV}$ and a more important decrease to zero is obtained at high frequency for select compositions. It should be reminded, however, that since the available $\mathrm{E}=\hbar \omega$ range is correlated with a finite $k$ range, the measured $\mathrm{g}(\omega)$ is slightly distorted and incomplete.

For elemental tellurium, the VDOS consists in an important contribution at low frequency $(\omega<10 \mathrm{meV})$ signaling also the possibility of low frequency (floppy) modes $(5 \mathrm{meV})$ that are typical of flexible glasses and glass-forming liquids [44]. As the Te content is reduced, this low frequency band tends to reduce in intensity and is driven by the progressive stiffening of the liquid structure due to the addition of $\mathrm{Ge} / \mathrm{Ga}$ cross links. Simultaneously, a second broad band centered around $20 \mathrm{meV}$ builds up with the concentration $x$.

The partial contribution to the VDOS (Fig. 13) indicates in fact that Ge atoms contribute to a broad band centered at 20$25 \mathrm{meV}$ [Fig. 13(b)], whereas the dominant contribution at low frequency continues to be mostly originated from Te atoms, this statement being valid even at smaller Te content [e.g.,
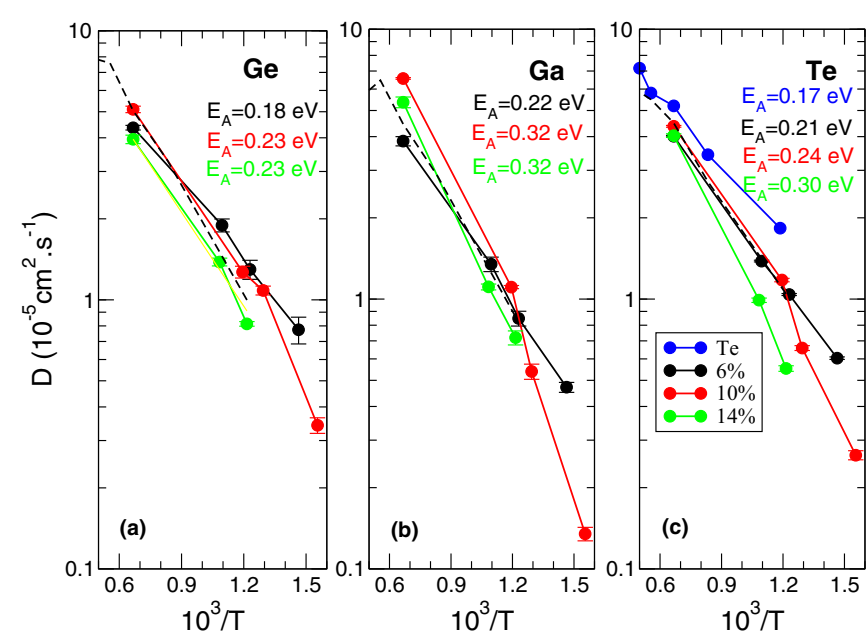

FIG. 14. Calculated diffusion constant for Ge (a), Ga (b), and $\mathrm{Te}$ (c) atoms in Ge-Ga-Te liquids with different compositions. The diffusivity of elemental Te is also shown [14]. The broken lines correspond to diffusivities of $\mathrm{Ge}_{14} \mathrm{Sb}_{14} \mathrm{Te}_{72}$ [14] and serve for comparison with $\mathrm{Ge}_{14} \mathrm{Ga}_{14} \mathrm{Te}_{72}$ (green curves). Activation energies $E_{A}$ are given for each species and composition. The errors have been obtained by calculating the standard deviation of the diffusion data.

$\mathrm{Ge}_{14} \mathrm{Ga}_{14} \mathrm{Te}_{72}$, Fig. 13(a)]. Finally, Ga atoms mostly contribute to the broad band at 20-30 meV [Fig. 13(c)].

\section{DYNAMIC PROPERTIES}

Results of this section are extracted only from the molecular dynamics simulations. From the calculated mean-square displacement of an atom of type $\alpha$ given by

$$
\left\langle r^{2}(t)\right\rangle=\frac{1}{N_{\alpha}} \sum_{i=1}^{N_{\alpha}}\left\langle\left|\mathbf{r}_{i}(t)-\mathbf{r}_{i}(0)\right|^{2}\right\rangle,
$$

one can extract the dependence of $\left\langle r^{2}(t)\right\rangle$ in the long time behavior where the dynamics becomes diffusive. $N_{\alpha}$ represents the number of atoms of a given species $\alpha$. Using the Einstein relation at long times $\lim _{t \rightarrow \infty}\left\langle r^{2}(t)\right\rangle / 6 t=D$, the diffusion constants $D$ for various species can be calculated from the mean square displacement as a function of composition and temperature. Here, we have used additional temperatures $(1500 \mathrm{~K}, 913 \mathrm{~K}, 773 \mathrm{~K}$, and $673 \mathrm{~K})$ that have been accumulated for the purpose of the dynamic study.

\section{A. Diffusivities}

Corresponding results are represented in Fig. 14 for the different compositions, and the data exhibit an Arrheniuslike dependence $\exp \left[-E_{A} / T\right]$ as already found for a variety of oxide or chalcogenide melts [45-48]. A fit using such a functional leads to values for the activation energies $E_{A}$ that are in the range of about $0.17-0.30 \mathrm{eV}$ depending on the composition, and a global increase of $E_{A}$ with $\mathrm{Ge} / \mathrm{Ga}$ content $x$ is obtained. These values are compatible with those determined numerically for, e.g., $\mathrm{Ge}_{2} \mathrm{Sb}_{2} \mathrm{Te}_{5}$ and for this composition activation energies were found to be of about $0.30 \mathrm{eV}, 0.26 \mathrm{eV}$, and $0.29 \mathrm{eV}$ for germanium, antimony, and tellurium, respectively [14]. The present numbers compare also 
well with those determined in other typical telluride liquids such as simulated GeTe $\left(E_{A}=0.30 \mathrm{eV}\right.$ and $E_{A}=0.32 \mathrm{eV}$ for Ge and Te, respectively [49]), viscosity determined $E_{A}$ in $\mathrm{Ge}_{2} \mathrm{Sb}_{2} \mathrm{Te}_{5}\left(0.266 \mathrm{eV}\right.$ [50]) or $\mathrm{Sb}_{4} \mathrm{Te}(0.30 \mathrm{eV}$ [51]), this comparison being especially valid when liquids with a similar degree of connectivity are being compared. One also remarks from Fig. 14 that activation energies for $\mathrm{Ge}$ atoms are smaller and lead therefore to lower energy barriers for diffusion and, possibly, crystallization kinetics.

The addition of cross-linking $\mathrm{Ge}$ and $\mathrm{Ga}$ atoms leads to a global reduction of the dynamics, this being true for all species, as detected from the decrease of, e.g., $D_{\mathrm{Ge}}$ from $(1.29 \pm$ $0.11) \times 10^{-5} \mathrm{~cm}^{2} \mathrm{~s}^{-1}$ to $(0.81 \pm 0.02) \times 10^{-5} \mathrm{~cm}^{2} \mathrm{~s}^{-1}$ between the $6 \%$ and the $14 \%$ alloys at $823 \mathrm{~K}$. One furthermore remarks that at low temperature (e.g., $843 \mathrm{~K}$ or $10^{3} / T=$ 1.19) $\mathrm{Ga}$ diffusivities are systematically smaller than the $\mathrm{Ge}$ counterparts (e.g., $D_{\mathrm{Ge}}=0.34 \times 10^{-5} \mathrm{~cm}^{2} \mathrm{~s}^{-1}$ and $D_{\mathrm{Ga}}=$ $0.13 \times 10^{-5} \mathrm{~cm}^{2} \mathrm{~s}^{-1}$ at $673 \mathrm{~K}$ ), a tendency that one might want to relate to the increased tetrahedral character of $\mathrm{Ga}$ (Table V). In fact, tetrahedral chalcogenide liquids (e.g., Ge-Se [52]) have diffusivities that are reduced and about of the order of $10^{-5} \mathrm{~cm}^{2} \mathrm{~s}^{-1}$ at higher temperatures $(1050 \mathrm{~K})$ but with activation energies that are substantially larger $(0.4-0.7 \mathrm{eV})$, a result that has been also recovered for tetrahedral oxide melts [47]. A comparison with the parent liquid $\mathrm{Ge}_{14} \mathrm{Sb}_{14} \mathrm{Te}_{72}$ (broken lines in Fig. 14) furthermore shows that the substitution of antimony by Ga atoms impacts essentially the low temperature dynamics of Te atoms because one has $D_{\mathrm{Sb}} \simeq D_{\mathrm{Ga}}$ and $\mathrm{Ge}$ diffusivities that are nearly equal between the two liquids.

\section{B. Relaxation properties and fragility}

In order to access to relaxation properties, we calculate the intermediate scattering function $F_{s}(k, t)$ which permits us to follow the decay of density fluctuations with time in Fourier space. It is a useful property for the investigation of relaxation properties [53]. From MD simulations and the positions $\mathbf{r}_{j}(t)$ of the atoms, the self intermediate scattering function $F_{s}^{\alpha}(k, t)$ for the species $\alpha$ can be calculated and is equal to:

$$
F_{s}^{\alpha}(k, t)=\frac{1}{N_{\alpha}} \sum_{j=1}^{N_{\alpha}} \exp \left(i \mathbf{k} \cdot\left(\mathbf{r}_{j}(t)-\mathbf{r}_{j}(0)\right)\right) .
$$

In Fig. 15, we represent time dependence of the Ga-based $F_{s}(k, t)$ for the three compositions of interest at the wave vectors corresponding to $k=k_{\max }=2.09 \AA^{-1}$, i.e., the position of the principal peak in the static structure factor $S(k)$ (Fig. 2). Results indicate the usual temperature dependence of the intermediate scattering function which shifts to longer times as the temperature is decreased (red curves), i.e., more time is needed to reach thermal equilibrium defined by $F_{s}(k, t)=0$. At high temperature $(1500 \mathrm{~K}), F_{s}(k, t)$ decreases within $1 \mathrm{ps}$ and the behavior exhibits a single Debye-like exponential decay. As the system becomes more viscous and exhibits a progressive glassy behavior $\left(673 \mathrm{~K}\right.$ corresponds to $T_{g} / T=0.64$ with $T_{g}=428 \mathrm{~K}$ [11]), a typical $\beta$-relaxation plateau builds up [53], and this plateau extends to longer times so that the simulation time ( 34 ps for the represented $\mathrm{Ge}_{10} \mathrm{Ga}_{10} \mathrm{Te}_{80}$ liquid, dotted red curve) does not permit us to obtain the full decay of $F_{s}(k, t)$ and signals an increasing glassy behavior. The

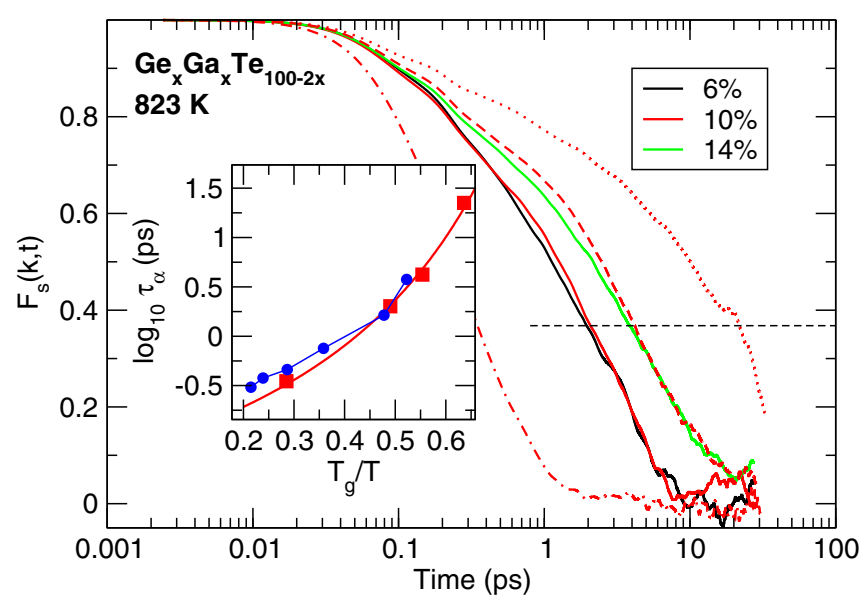

FIG. 15. Calculated Ga-based intermediate scattering function $F_{s}(k, t)$ in Ge-Ga-Te liquids at $823 \mathrm{~K}$. Additional red curves correspond to $\mathrm{Ge}_{10} \mathrm{Ga}_{10} \mathrm{Te}_{80}$ liquids at $1500 \mathrm{~K}$ (dot-dashed), $773 \mathrm{~K}$ (broken), and $673 \mathrm{~K}$ (dotted). The horizontal line corresponds to $1 / e$ and serves for the estimation of the relaxation time $\tau_{\alpha}$. The inset represents $\tau_{\alpha}$ for $\mathrm{Ge}_{10} \mathrm{Ga}_{10} \mathrm{Te}_{80}$ (red squares) in a reduced Angell plot where $T_{g}$ has been taken as $428 \mathrm{~K}$ [11]. The red curve is a VFT fit [Eq. (8)] to the Ge-Ga-Te data using $\log _{10} \tau_{\infty}=-13.12$ and $\mathcal{M}=139$. Blue symbols correspond to data for $\mathrm{Ge}_{2} \mathrm{Sb}_{2} \mathrm{Te}_{5}$ [14].

effect of composition appears to be weak for all species (Ge, $\mathrm{Te}$, not shown) between $\mathrm{Ge}_{6} \mathrm{Ga}_{6} \mathrm{Te}_{88}$ and $\mathrm{Ge}_{10} \mathrm{Ga}_{10} \mathrm{Te}_{80}$ and corresponding relaxation times $\tau_{\alpha}$ are found to be of the order of $1-2 \mathrm{ps}$ for the temperature $823 \mathrm{~K}, \tau_{\alpha}$ being obtained by setting $F_{s}\left(k, \tau_{\alpha}\right)=1 / e$ as also used in the literature [54,55].

For the particular $\mathrm{Ge}_{10} \mathrm{Ga}_{10} \mathrm{Te}_{80}$ liquid, we can then represent $\tau_{\alpha}$ in a reduced representation (i.e., as a function of $\left.T_{g} / T\right)$ using $T_{g}=428 \mathrm{~K}$ [11] that has been measured for the parent system $\mathrm{Ge}_{11} \mathrm{Ga}_{11} \mathrm{Te}_{78}$ [11]. Results (inset of Fig. 15) show that the relaxation time is similar to the one obtained for $\mathrm{Ge}_{2} \mathrm{Sb}_{2} \mathrm{Te}_{5}$ [14], the latter having a similar glass transition temperature (430 K [56]). A useful and popular means to quantify simply the temperature dependence and the slowing down of the relaxation with decreasing temperature uses the fragility index $\mathcal{M}$ that is defined as [57]:

$$
\mathcal{M} \equiv\left[\frac{d \log _{10} \tau_{\alpha}}{d T_{g} / T}\right]_{T=T_{g}} .
$$

Large $\mathcal{M}$ (termed as fragile liquids) typical of organic liquids [53] or certain tellurides $[14,16,17]$ lead to a curvature in the plot $\left(\log _{10} \tau_{\alpha}\right.$ with $\left.T_{g} / T\right)$ and induce a very rapid variation of $\tau_{\alpha}$ in the vicinity of the glass transition unless some more subtle changes (fragile to strong transitions) occur close to the melting point $[16,58]$. Here, $T_{g}$ is the glass (reference) temperature at which one usually has $\tau_{\alpha}=100 \mathrm{~s}$ or a viscosity of $\eta=10^{12}$ $\mathrm{Pa}$ s. Using the definition of Eq. (7), the fragility index can be used as a parameter for a Vogel-Fulcher-Tamman (VFT) form describing the temperature behavior of the relaxation time [53]:

$$
\log _{10} \tau_{\alpha}(T)=\log _{10} \tau_{\infty}+\frac{\left(2-\log _{10} \tau_{\infty}\right)^{2}}{\mathcal{M}\left(T / T_{g}-1\right)+\left(2-\log _{10} \tau_{\infty}\right)},
$$


where it is assumed that $\tau_{\alpha}$ at $T_{g}$ is equal to $100 \mathrm{~s}$, and $\tau_{\infty}$ represents the relaxation time at very high (infinite) temperature, supposed to be of the order of atomic vibrations $\left(\log _{10} \tau_{\infty}=-13\right.$ ). A fit to the data of $\mathrm{Ge}_{10} \mathrm{Ga}_{10} \mathrm{Te}_{80}$ (red curve in the inset of Fig. 15) leads to $\log _{10} \tau_{\infty}=-13.12$ and to $\mathcal{M}=139$ if one uses the reported glass transition temperature [11].

The obtained value indicates that the fragility of Ge-Ga-Te liquids is large, at least at high temperature. These findings are in line with the recent reported high fragility of $\mathrm{Ge}_{2} \mathrm{Sb}_{2} \mathrm{Te}_{5}$ $(\mathcal{M}=129$ [14]) that facilitates the physical features involved in data storage. A high fragility leads to low activation barriers for diffusion [Fig. 14(b)] which are of the order of $0.25 \mathrm{eV}$ in several other phase change materials $\left(\mathrm{Ge}_{2} \mathrm{Sb}_{2} \mathrm{Te}_{5}\right.$ [50], AIST [15], or $\mathrm{Sb}_{4} \mathrm{Te}$ [51]). This ensures fast crystallization at high temperature given that the crystal growth velocity is directly proportional to the diffusivity $\mathrm{D}(\mathrm{T})$.

\section{CONCLUSIONS}

Ge-Te based materials represent a very promising class of materials with important optoelectronic applications once additional elements such as $\mathrm{Sb}$ or $\mathrm{Ga}$ are incorporated. However, specific properties that need to be continuously optimized with chemical composition depend crucially on the underlying liquid or amorphous structure. The description of structural properties of such tellurides needs, therefore, to be completed using various techniques.

Here we have focused on the structural, vibrational, and dynamic properties of three compositions in the liquid Ge-GaTe ternary by combining molecular dynamics simulations, neutron diffraction, and inelastic neutron scattering experiments. We have also considered simulations [34] and experiments [25] from elemental Te. The study reveals that both the experimental pair correlation function in real space, and the structure factor in reciprocal space can be reproduced from molecular simulations, the degree of accuracy of the calculated functions with the experimental counterpart evolving with $\mathrm{Ge} / \mathrm{Ga}$ content. Such simulated models then provide access to additional information on structure such as fourfold Ge and $\mathrm{Ga}$ atoms but a coordination number for Te that is substantially higher than what would be expected from the octet rule. For all compositions, we also found that the structure of these Ge-Ga-Te liquids is made of a majority of germanium in a defective octahedral geometry $(76.2-83.4 \%)$, whereas Ga is more likely in tetrahedral configuration $(\simeq 75 \%)$, the tetrahedral nature of the liquid increasing with $\mathrm{Ge} / \mathrm{Ga}$ content as determined from angular topological constraints. Such features differ from the picture drawn in Ge-Sb-Te liquids which are predominantly octahedral-like. The analysis of the vibrational properties furthermore shows a correct agreement between simulated and experimental data, this statement being true for all studied compositions. Finally, we investigate the liquid dynamics and find that Ge-Ga-Te liquids behave every similarly to $\mathrm{Ge}-\mathrm{Sb}$-Te liquids once their respective relaxation times $\tau_{\alpha}$ have been represented in an appropriate Angell representation [57], i.e., $\tau_{\alpha}\left(T_{g} / T\right)$. The corresponding fragility $\mathcal{M}=139$ for $\mathrm{Ge}_{10} \mathrm{Ga}_{10} \mathrm{Te}_{80}$ is found to be comparable to the one recently determined for $\mathrm{Ge}_{2} \mathrm{Sb}_{2} \mathrm{Te}_{5}$ [14].

It would be interesting to probe how the structure evolves with composition at lower temperatures and in the amorphous phase, as recently investigated [59]. Work in this direction is in progress.

\section{ACKNOWLEDGMENTS}

The authors thank Jean-Yves Raty for useful and stimulating discussions. Support from Agence Nationale de la Recherche (ANR) (Grant No. ANR-11-BS08-0012) is gratefully acknowledged. GENCI (Grand Equipement National de Calcul Intensif), CINaM and Plateforme Roméo de l'Université de Reims Champagne Ardenne are acknowledged for supercomputing access.
[1] Phase Change Materials and Applications, edited by S. Raoux and M. Wuttig (Springer, Berlin, 2008).

[2] L. van Pieterson, M. H. R. Lankhorst, M. van Schijndel, A. E. T. Kuiper, and J. H. J. Rosen, J. Appl. Phys. 97, 083520 (2005).

[3] A. Wilhelm, C. Boussard-Plédel, Q. Coulombier, J. Lucas, B. Bureau, and P. Lucas, Adv. Mater. 19, 3796 (2007).

[4] V. S. Shiryaev, J. L. Adam, X. H. Zhang, C. Boussard-Plédel, J. Lucas, and M. F. Churbanov, J. Non-Cryst. Solids 336, 113 (2004).

[5] C. Vigreux-Bercovici, E. Bonhomme, A. Pradel, J. E. Broquin, L. Labadie, and P. Kern, Appl. Phys. Lett. 90, 011110 (2007).

[6] X. H. Zhang, L. Calvez, V. Seznec, H. L. Ma, S. Danto, P. Houizat, C. Boussard-Plédel, and J. Lucas, J. Non-Cryst. Solids 352, 2411 (2006).

[7] M. A. Popescu, Non-Crystalline Chalcogenides (Kluwer Academic Ed., Dordrecht, 2000).

[8] S. Danto, P. Houizot, C. Boussard-Pledel, X.-H. Zhang, F. Smektala, and J. Lucas, Adv. Funct. Mater. 16, 1847 (2006).
[9] C. Vigreux-Bercovici, E. Bonhomme, and A. Pradel, J. NonCryst. Solids 353, 1388 (2007).

[10] I. Voleská, J. Akola, P. Jóvári, J. Gutwirth, T. Wágner, Th. Vasileiadis, S. N. Yannopoulos, and R. O. Jones, Phys. Rev. B 86, 094108 (2012).

[11] R. Golovchak, L. Calvez, B. Bureau, and H. J. Jain, J. Chem. Phys. 139, 054508 (2013).

[12] P. Jovari, I. Kaban, B. Bureau, A. Wilhelm, P. Lucas, B. Beuneu, and D. A. Zajac, J. Phys. Condens. Matter 22, 404207 (2010).

[13] R. Svoboda, D. Stríteský, Z. Zmrhalová, D. Brandová, and J. Málek, J. Non-Cryst. Solids 445-446, 7 (2016).

[14] H. Flores-Ruiz and M. Micoulaut, J. Chem. Phys. 148, 034502 (2018).

[15] W. Zhang, I. Ronneberger, P. Zalden, M. Xu, M. Salinga, M. Wuttig, and R. Mazzarello, Sci. Rep. 4, 6529 (2014).

[16] S. Wei, G. J. Coleman, P. Lucas, and C. A. Angell, Phys. Rev. Appl. 7, 034035 (2017).

[17] S. Wei, P. Lucas, and C. A. Angell, J. Appl. Phys. 118, 034903 (2015). 
[18] A. Piarristeguy, M.-V. Coulet, M. R. Johnson, and G. Cuello, ILL experimental report 6-03-414 (2011).

[19] H. E. Fischer, G. J. Cuello, P. Palleau, D. Feltin, A. C. Barnes, Y. S. Badyal, and J. M. Simonson, Appl. Phys. A 74, s160 (2002).

[20] CORRECT: A correction program for neutron diffraction data, Version 2.4, M. A. Howe, R. L. McGreevy, and P. Zetterstrm (1998).

[21] Y. Tsuchiya, J. Phys. Soc. Jpn. 60, 227 (1991).

[22] LAMP, the Large Array Manipulation Program, http://www.ill.fr/data_treat/lamp/the_lamp-book.

[23] H. Flores-Ruiz, M. Micoulaut, M.-V. Coulet, A. A. Piarristeguy, M. R. Johnson, G. J. Cuello, and A. Pradel, Phys. Rev. B 92, 134205 (2015).

[24] R. Car and M. Parrinello, Phys. Rev. Lett. 55, 2471 (1985).

[25] J. Akola, R. O. Jones, S. Kohara, T. Usuki, and E. Bychkov, Phys. Rev. B 81, 094202 (2010).

[26] J. Kalikka, J. Akola, and R. O. Jones, J. Phys. Condens. Matter 25, 115801 (2013).

[27] M. Micoulaut, R. Vuilleumier, and C. Massobrio, Phys. Rev. B 79, 214205 (2009).

[28] A. Bouzid, C. Massobrio, M. Boero, G. Ori, K. Sykina, and E. Furet, Phys. Rev. B 92, 134208 (2015).

[29] A. Bouzid, G. Ori, M. Boero, E. Lampin, and C. Massobrio, Phys. Rev. B 96, 224204 (2017).

[30] N. Trouiller and J. L. Martins, Phys. Rev. B 43, 1993 (1991).

[31] S. Nosé, Mol. Phys. 52, 255 (1984); W. G. Hoover, Phys. Rev. A 31, 1695 (1985).

[32] A. Menelle, R. Bellisent, and A. M. Flank, Europhys. Lett. 4, 705 (1987).

[33] C. Steimer, M. V. Coulet, W. Welnic, H. Dieker, R. Detemple, C. Bichara, B. Beuneu, J. P. Gaspard, and M. Wuttig, Adv. Mater. 20, 4535 (2008).

[34] M. Micoulaut, M.-V. Coulet, A. Piarristeguy, M. R. Johnson, G. J. Cuello, C. Bichara, J.-Y. Raty, H. Flores-Ruiz, and A. Pradel, Phys. Rev. B 89, 174205 (2014).

[35] A. Zeidler, K. Wezka, R. F. Rowlands, D. A. J. Whittaker, P. S. Salmon, A. Polidori, J. W. E. Drewitt, S. Klotz, H. E. Fischer, M. C. Wilding, C. L. Bull, M. G. Tucker, and M. Wilson, Phys. Rev. Lett. 113, 135501 (2014).

[36] A. C. Wright, J. Non-Cryst. Solids 159, 264 (1993).

[37] S. Tao, J. P. Perdew, V. N. Staroverov, and G. E. Scuseria, Phys. Rev. Lett. 91, 146401 (2003).
[38] Landolt-Börnstein, in New Series I/16A, edited by H. Schopper (Springer, Berlin, 2000).

[39] A. Piarristeguy, M. Micoulaut, R. Escalier, P. Jóvári, I. Kaban, J. Van Eijk-Steiner, J. Luckas, S. Ravindren, P. Boolchand, and A. Pradel, J. Chem. Phys. 143, 074502 (2015).

[40] M. Micoulaut, K. Gunasekera, S. Ravindren, and P. Boolchand, Phys. Rev. B 90, 094207 (2014).

[41] M. Micoulaut, A. Kachmar, M. Bauchy, S. Le Roux, C. Massobrio, and M. Boero, Phys. Rev. B 88, 054203 (2013).

[42] K. Gunasekera, P. Boolchand, and M. Micoulaut, J. Appl. Phys. 115, 164905 (2014).

[43] M. Micoulaut, Adv. Physics X 1, 147 (2016).

[44] W. A. Kamitakahara, R. L. Cappelletti, P. Boolchand, B. Halfpap, F. Gompf, D. A. Neumann, and H. Mutka, Phys. Rev. B 44, 94 (1991).

[45] M. Bauchy, M. Micoulaut, M. Boero, and C. Massobrio, Phys. Rev. Lett. 110, 165501 (2013).

[46] M. Micoulaut, Y. Guissani, and B. Guillot, Phys. Rev. E 73, 031504 (2006).

[47] J. Horbach and W. Kob, Phys. Rev. B 60, 3169 (1999).

[48] M. Bauchy, A. Kachmar, and M. Micoulaut, J. Chem. Phys. 141, 194506 (2014).

[49] J. Liu, X. Xu, L. Brush, and M. P. Anantram, J. Appl. Phys. 115, 023513 (2014).

[50] M. Schumacher, H. Weber, P. Jóvári, Y. Tsuchiya, T. G. A Youngs, I. Kaban, and R. Mazzarello, Sci. Rep. 6, 27434 (2016).

[51] F. W. Herwig, Z. Metallkd 83, 35 (1992).

[52] C. Yildirim, J.-Y. Raty, and M. Micoulaut, J. Chem. Phys. 144, 224503 (2016).

[53] M. Micoulaut, Rep. Progr. Phys. 79, 066504 (2016).

[54] W. Kob and H. C. Andersen, Phys. Rev. E 52, 4134 (1995).

[55] N. A. Morgan and F. J. Spera, Geochimica et Cosmochimica Acta 65, 4019 (2001).

[56] J. Orava, L. Greer, B. Gholipour, D. W. Hewak, and C. E. Smith, Nat. Mater. 11, 279 (2012).

[57] C. A. Angell, Science 267, 1924 (1995).

[58] C. Zhang, L. Hu, Y. Yue, and J. C. Mauro, J. Chem. Phys. 133, 014508 (2010).

[59] Z. Chaker, G. Ori, M. Boero, C. Massobrio, E. Furet, and A. Bouzid, J. Non-Cryst. Solids (2018), doi: 10.1016/j.jnoncrysol.2018.03.039. 